\title{
Canonical characters on quasi-symmetric functions and bivariate Catalan numbers*
}

\author{
Marcelo Aguiar \\ Department of Mathematics \\ Texas A\&M University \\ College Station, TX 77843, USA \\ maguiar@math . tamu . edu \\ Samuel K. Hsiao \\ Department of Mathematics \\ University of Michigan \\ Ann Arbor, MI 48109, USA \\ shsiao@umich.edu
}

Submitted: Sep 4, 2004; Accepted: Dec 31, 2004; Published: Feb 21, 2005

Mathematics Subject Classifications: 05A15, 05E05, 16W30, 16W50.

Keywords: Hopf algebra, character, quasi-symmetric function, central binomial coefficient, Catalan number, bivariate Catalan number, peak of a permutation.

\begin{abstract}
Every character on a graded connected Hopf algebra decomposes uniquely as a product of an even character and an odd character (Aguiar, Bergeron, and Sottile, math.CO/0310016). We obtain explicit formulas for the even and odd parts of the universal character on the Hopf algebra of quasi-symmetric functions. They can be described in terms of Legendre's beta function evaluated at half-integers, or in terms of bivariate Catalan numbers:

$$
C(m, n)=\frac{(2 m) !(2 n) !}{m !(m+n) ! n !} .
$$

Properties of characters and of quasi-symmetric functions are then used to derive several interesting identities among bivariate Catalan numbers and in particular among Catalan numbers and central binomial coefficients.
\end{abstract}

*Work supported in part by NSF grant DMS-0302423 and by the NSF Postdoctoral Research Fellowship. We benefited from discussions with Ira Gessel and from the expertise of François Jongmans, who generously helped us search the 19th century literature in pursuit of a hard-to-find article by Catalan. We also thank the referees for interesting remarks and suggestions.

THE ELECTRONiC JOURNAL OF COMBINATORICS 11(2) (2005), \#R15 


\section{Introduction}

The numbers

$$
C(m, n):=\frac{(2 m) !(2 n) !}{m !(m+n) ! n !}=\frac{\left(\begin{array}{c}
2 m \\
m
\end{array}\right)\left(\begin{array}{c}
2 n \\
n
\end{array}\right)}{\left(\begin{array}{c}
m+n \\
n
\end{array}\right)}
$$

appeared in work of Catalan, [4, pp. 14-15], [5, p. 207], [6, Sections CV and CCXIV], [7, pp. 110-113], von Szily [25, pp. 89-91], Riordan [19, Chapter 3, Exercise 9, p. 120], and recent work of Gessel [12]. We call them bivariate Catalan numbers. They are integers (and except for $C(0,0)=1$, they are all even). Special cases include the central binomial coefficients and the Catalan numbers:

$$
C(0, n)=\left(\begin{array}{c}
2 n \\
n
\end{array}\right) \text { and } \frac{1}{2} C(1, n)=\frac{1}{n+1}\left(\begin{array}{c}
2 n \\
n
\end{array}\right) .
$$

In turn, the bivariate Catalan numbers are special cases of the super ballot numbers of Gessel [12].

The algebra $\mathcal{Q}$ Sym of quasi-symmetric functions was introduced in earlier work of Gessel [11] as a source of generating functions for Stanley's P-partitions [20]; since then, the literature on the subject has become vast. The linear bases of $\mathcal{Q} S y m$ are indexed by compositions $\alpha$ of $n$. Two important bases are given by the monomial and fundamental quasi-symmetric functions $M_{\alpha}$ and $F_{\alpha}$; for more details, see [11], 17, Chapter 4], or [21, Section 7.19].

In [2], an important universal property of $\mathcal{Q} S y m$ was derived. Consider the functional $\zeta: \mathcal{Q}$ Sym $\rightarrow \mathbb{k}$ obtained by specializing one variable of a quasi-symmetric function to 1 and all other variables to 0. On the monomial and fundamental bases of $\mathcal{Q}$ Sym, this functional is given by

$$
\zeta\left(M_{\alpha}\right)=\zeta\left(F_{\alpha}\right)= \begin{cases}1 & \text { if } \alpha=(n) \text { or }(), \\ 0 & \text { otherwise. }\end{cases}
$$

A character on a Hopf algebra $\mathcal{H}$ is a morphism of algebras $\varphi: \mathcal{H} \rightarrow \mathbb{k}$ :

$$
\varphi(a b)=\varphi(a) \varphi(b), \quad \varphi(1)=1 .
$$

$\mathcal{Q}$ Sym is a graded connected Hopf algebra and the functional $\zeta$ is a character on $\mathcal{Q}$ Sym . The universal property states that given any graded connected Hopf algebra $\mathcal{H}$ and a character $\varphi: \mathcal{H} \rightarrow \mathbb{k}$, there exists a unique morphism of graded Hopf algebras $\Phi: \mathcal{H} \rightarrow$ $\mathcal{Q}$ Sym making the following diagram commutative [2, Theorem 4.1]:

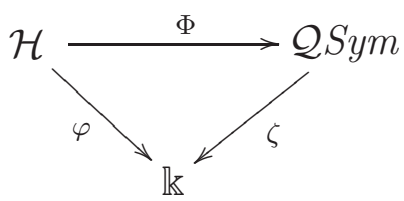

For this reason, we refer to $\zeta$ as the universal character on $\mathcal{Q} S y m$. There are other characters on $\mathcal{Q}$ Sym canonically associated to $\zeta$ that are of interest to us. In spite of the 
simple definition of $\zeta$, these characters encompass important combinatorial information. Some of these were explicitly described and studied in [2], and shown to be closely related to a Hopf subalgebra of $\mathcal{Q}$ Sym introduced by Stembridge [23, to the generalized Dehn-Sommerville relations, and to other combinatorial constructions. Other canonical characters, less easy to describe but of a more fundamental nature, are the object of this paper.

We review other relevant background and constructions from [2].

Let $\mathcal{H}$ be an arbitrary Hopf algebra. The convolution product of two linear functionals $\rho, \psi: \mathcal{H} \rightarrow \mathbb{k}$ is

$$
\mathcal{H} \stackrel{\Delta}{\longrightarrow} \mathcal{H} \otimes \mathcal{H} \stackrel{\rho \otimes \psi}{\longrightarrow} \mathbb{k} \otimes \mathbb{k} \stackrel{m}{\longrightarrow} \mathbb{k},
$$

where $\Delta$ is the coproduct of $\mathcal{H}$ and $m$ is the product of the base field. We denote the convolution product by $\rho \psi$. The set of characters on any Hopf algebra is a group under the convolution product. The unit element is $\epsilon: \mathcal{H} \rightarrow \mathbb{k}$, the counit map of $\mathcal{H}$. The inverse of a character $\varphi$ is $\varphi^{-1}:=\varphi \circ S$, where $S$ is the antipode of $\mathcal{H}$.

Suppose that $\mathcal{H}$ is graded, i.e., $\mathcal{H}=\oplus_{n \geq 0} \mathcal{H}_{n}$ and the structure maps of $\mathcal{H}$ preserve this decomposition. This means that $\mathcal{H}_{i} \cdot \mathcal{H}_{j} \subseteq \mathcal{H}_{i+j}, \Delta\left(\mathcal{H}_{n}\right) \subseteq \sum_{i+j=n} \mathcal{H}_{i} \otimes \mathcal{H}_{j}, 1 \in \mathcal{H}_{0}$, and $\epsilon\left(\mathcal{H}_{n}\right)=0$ for $n>0$. Then $\mathcal{H}$ carries a canonical automorphism defined on homogeneous elements $h$ of degree $n$ by $h \mapsto \bar{h}:=(-1)^{n} h$. If $\varphi$ is a functional on $\mathcal{H}$, we define a functional $\bar{\varphi}$ by $\bar{\varphi}(h)=\varphi(\bar{h})$. The functional $\varphi$ is said to be even if

$$
\bar{\varphi}=\varphi
$$

and it is said to be odd if it is invertible with respect to convolution and

$$
\bar{\varphi}=\varphi^{-1}
$$

Suppose now that $\mathcal{H}$ is graded and connected, i.e., $\mathcal{H}_{0}=\mathbb{k} \cdot 1$. One of the main results of [2] states that any character $\varphi$ on $\mathcal{H}$ decomposes uniquely as a product of characters

$$
\varphi=\varphi_{+} \varphi_{-}
$$

with $\varphi_{+}$even and $\varphi_{-}$odd [2, Theorem 1.5].

The main purpose of this paper is to obtain explicit descriptions for the canonical characters $\zeta_{+}$and $\zeta_{-}$of $\mathcal{Q}$ Sym. We find that the values of both characters are given in terms of bivariate Catalan numbers (up to signs and powers of 2). On the monomial basis, the values are Catalan numbers and central binomial coefficients (Theorem 3.2). On the fundamental basis, general bivariate Catalan numbers intervene (Theorem 5.11). The connection with Legendre's beta function is given in Remark 5.2. The proofs rely on a number of identities for these numbers, of which some are known and others are new. In turn, the general properties of even and odd characters imply further identities that these numbers must satisfy. We obtain in this way a large supply of identities for Catalan numbers and central binomial coefficients (Section 4) and for bivariate Catalan numbers (Sections 6 and 7). As one should expect, some of these identities may also be obtained by more standard combinatorial arguments, at least once one is confronted with them. 
Our methods, however, yield the identities without any previous knowledge of their form. We mention here four of the most representative among the identities we derive:

$$
\begin{gathered}
\sum_{\substack{\alpha<\beta \\
a_{1} \text { odd }}} \frac{(-1)^{k_{e}(\alpha)}}{4^{\left\lfloor k_{o}(\alpha) / 2\right\rfloor}}\left(\begin{array}{c}
2\left\lfloor k_{o}(\alpha) / 2\right\rfloor \\
\left.k_{o}(\alpha) / 2\right\rfloor
\end{array}\right)=0 ; \\
\sum_{j=0}^{h} C_{3}(j) C_{3}(h-j)=2 \sum_{j=0}^{h-1} C_{2}(j) C_{1}(h-1-j) \\
\sum_{\sigma \in S_{n}}(-1)^{p_{-}(\sigma)} C\left(p_{-}(\sigma),\lfloor n / 2\rfloor-p_{-}(\sigma)\right)=4^{\lfloor n / 2\rfloor} \\
2 C(r)=\frac{1}{4^{s}} C(r, s+1)+\sum_{j=1}^{s} \frac{1}{4^{j}} C(r+1, j) .
\end{gathered}
$$

In (14),$k_{e}(\alpha)$ and $k_{o}(\alpha)$ are the number of even parts and the number of odd parts of a composition $\alpha, \beta=\left(b_{1}, \ldots, b_{k}\right)$ is any fixed composition such that $k_{e}(\beta) \equiv 0$ and $b_{1} \equiv b_{k} \bmod 2$, and the sum is over those compositions $\alpha=\left(a_{1}, \ldots, a_{h}\right)$ whose first part is odd and which are strictly refined by $\beta$. The numbers $C_{i}(j)$ appearing in (40) are central Catalan numbers; see (39). In (51), $p_{-}(\sigma)$ denotes the number of interior peaks of the permutation $\sigma$; see Section 7. Equation (67) expresses a Catalan number in terms of bivariate Catalan numbers.

In Section 8 we derive explicit formulas for the even and odd characters entering in the decomposition of the inverse (with respect to convolution) of the universal character, and deduce some more identities, including (67).

We work over a field $\mathbb{k}$ of characteristic different from 2.

\section{Even and odd characters}

Let $\mathcal{H}$ be a graded connected Hopf algebra and $\varphi: \mathcal{H} \rightarrow \mathbb{k}$ a linear functional such that $\varphi(1)=1$ (this holds if $\varphi$ is a character). Let $\varphi_{n}$ denote the restriction of $\varphi$ to the homogeneous component of $\mathcal{H}$ of degree $n$. By assumption, $\varphi_{0}=\epsilon_{0}$, where $\epsilon$ is the counit of $\mathcal{H}$. This guarantees that $\varphi$ is invertible with respect to convolution: the inverse functional $\varphi^{-1}$ is determined by the recursion

$$
\left(\varphi^{-1}\right)_{n}=-\sum_{i=1}^{n} \varphi_{i}\left(\varphi^{-1}\right)_{n-i}
$$

with initial condition $\left(\varphi^{-1}\right)_{0}=\epsilon_{0}$. The right hand side denotes the convolution product of $\varphi_{i}$ and $\left(\varphi^{-1}\right)_{n-i}$, viewed as functionals on $\mathcal{H}$ which are zero on degrees different from $i$ and $n-i$, respectively.

Lemma 2.1. Let $\mathcal{H}$ be a graded connected Hopf algebra and $\varphi: \mathcal{H} \rightarrow \mathbb{k}$ a linear functional such that $\varphi(1)=1$. There are unique linear functionals $\rho, \psi: \mathcal{H} \rightarrow \mathbb{k}$ such that 
(a) $\rho(1)=\psi(1)=1$,

(b) $\rho$ is even and $\psi$ is odd,

(c) $\varphi=\rho \psi$.

Moreover, if $\varphi$ is a character then so are $\rho$ and $\psi$.

Proof. Items (a), (b), and (c) can be derived as in the proof of [2, Theorem 1.5], while [2, Proposition 1.4] guarantees that if $\varphi$ is a character then so are $\rho$ and $\psi$.

In this situation, we write $\varphi_{+}:=\rho$ and $\varphi_{-}:=\psi$ and refer to them as the even part and the odd part of $\varphi$. According to the results cited above, $\varphi_{+}$is uniquely determined by the recursion

$$
(-1)^{n} \varphi_{n}=2\left(\varphi_{+}\right)_{n}+\left(\varphi^{-1}\right)_{n}+\sum_{\substack{i+j+k=n \\ 0 \leq i, j, k<n}}\left(\varphi_{+}\right)_{i}\left(\varphi^{-1}\right)_{j}\left(\varphi_{+}\right)_{k}
$$

and $\varphi_{-}$by

$$
\left(\varphi_{-}\right)_{n}=\varphi_{n}-\sum_{i=1}^{n}\left(\varphi_{+}\right)_{i}\left(\varphi_{-}\right)_{n-i}
$$

with initial conditions $\left(\varphi_{+}\right)_{0}=\left(\varphi_{-}\right)_{0}=\epsilon_{0}$.

Lemma 2.2. Suppose $\mathcal{H}$ and $\mathcal{K}$ are graded connected Hopf algebras, $\varphi: \mathcal{H} \rightarrow \mathbb{k}$ and $\psi: \mathcal{K} \rightarrow \mathbb{K}$ are characters, and $\Phi: \mathcal{H} \rightarrow \mathcal{K}$ is a morphism of graded Hopf algebras such that

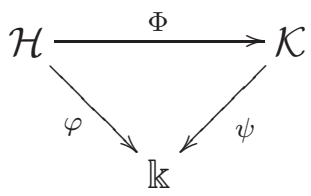

commutes. Then the diagrams
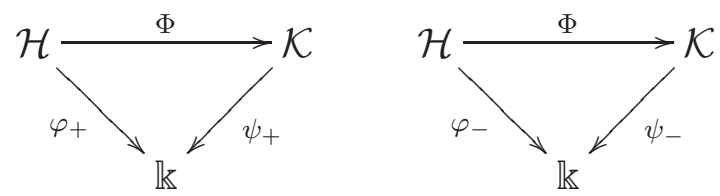

commute as well.

Proof. Composition with $\Phi$ gives a morphism from the character group of $\mathcal{K}$ to the character group of $\mathcal{H}$ which preserves the canonical involution $\varphi \mapsto \bar{\varphi}$. Thus $\psi=\psi_{+} \psi_{-}$ implies $\psi \circ \Phi=\left(\psi_{+} \circ \Phi\right)\left(\psi_{-} \circ \Phi\right), \psi_{+} \circ \Phi$ is even, and $\psi_{-} \circ \Phi$ is odd. By uniqueness in Lemma 2.1. $\psi_{+} \circ \Phi=\varphi_{+}$and $\psi_{-} \circ \Phi=\varphi_{-}$. 
When $\mathcal{H}=\mathcal{Q}$ Sym and $\varphi=\zeta$ is the universal character (2), we refer to $\zeta_{+}$and $\zeta_{-}$as the canonical characters of $\mathcal{Q}$ Sym.

Let $\rho$ and $\psi$ be arbitrary characters on $\mathcal{Q}$ Sym. For later use, we describe the convolution product $\rho \psi$ explicitly. Given a composition $\alpha=\left(a_{1}, \ldots, a_{k}\right)$ of a positive integer $n$ and $0 \leq i \leq n$, let $\alpha_{i}=\left(a_{1}, \ldots, a_{i}\right)$ and $\alpha^{i}=\left(a_{i+1}, \ldots, a_{k}\right)$. We agree that $\alpha_{0}=\alpha^{k}=()$ (the empty composition). The coproduct of $\mathcal{Q} S y m$ is

$$
\Delta\left(M_{\alpha}\right)=\sum_{i=0}^{k} M_{\alpha_{i}} \otimes M_{\alpha^{i}},
$$

where $M_{()}$denotes the unit element $1 \in \mathcal{Q}$ Sym. It follows that

$$
(\rho \psi)\left(M_{\alpha}\right)=\sum_{i=0}^{k} \rho\left(M_{\alpha_{i}}\right) \psi\left(M_{\alpha^{i}}\right) .
$$

The counit is

$$
\epsilon\left(M_{\alpha}\right)= \begin{cases}1 & \text { if } \alpha=() \\ 0 & \text { otherwise }\end{cases}
$$

\section{The canonical characters of $\mathcal{Q} S y m$ on the monomial basis}

For any non-negative integer $m$, let

$$
B(m):=C(0, m)=\left(\begin{array}{c}
2 m \\
m
\end{array}\right) \text { and } C(m):=\frac{1}{2} C(1, m)=\frac{1}{m+1}\left(\begin{array}{c}
2 m \\
m
\end{array}\right) ;
$$

these are the central binomial coefficients and the Catalan numbers.

Lemma 3.1. For any non-negative integer $m$,

$$
\begin{aligned}
B(m) & =2 \sum_{i=1}^{m} C(i-1) B(m-i), \\
2^{2 m} & =\sum_{i=0}^{m} B(i) B(m-i) .
\end{aligned}
$$

Proof. These are well-known identities. They appear in [14, Formulas (3.90) and (3.92)], and [19, Chapter 3, Exercise 9, p. 120, and Section 4.2, Example 2, p. 130]. For bijective proofs, see [9, Formulas (2) and (8)].

For a composition $\alpha$, let $|\alpha|$ denote the sum of the parts of $\alpha, k(\alpha)$ the number of parts of $\alpha, k_{e}(\alpha)$ the number of even parts of $\alpha$, and $k_{o}(\alpha)$ the number of odd parts of $\alpha$. Note that

$$
k_{o}(\alpha) \equiv|\alpha| \bmod 2 \text {. }
$$


Theorem 3.2. Let $\alpha=\left(a_{1}, \ldots, a_{k}\right)$ be a composition of a positive integer $n$. Then

$$
\begin{aligned}
& \zeta_{-}\left(M_{\alpha}\right)= \begin{cases}\frac{(-1)^{k_{e}(\alpha)}}{2^{2\left\lfloor k_{o}(\alpha) / 2\right\rfloor} C\left(0,\left\lfloor k_{o}(\alpha) / 2\right\rfloor\right)} & \text { if } a_{k} \text { is odd, } \\
0 & \text { if } a_{k} \text { is even; }\end{cases} \\
& \zeta_{+}\left(M_{\alpha}\right)= \begin{cases}\frac{(-1)^{k_{e}(\alpha)+1}}{2^{k_{o}(\alpha)}} C\left(1, k_{o}(\alpha) / 2-1\right) & \text { if } a_{1} \text { and } a_{k} \text { are odd and } n \text { is even, } \\
1 & \text { if } \alpha=(n) \text { and } n \text { is even, } \\
0 & \text { otherwise. }\end{cases}
\end{aligned}
$$

We also have $\zeta_{-}(1)=\zeta_{+}(1)=1$.

Proof. Let $\rho, \psi: \mathcal{Q}$ Sym $\rightarrow \mathbb{k}$ be the linear maps defined by the proposed formula for $\zeta_{+}$ and $\zeta_{-}$, respectively. According to Lemma 2.1, to conclude $\rho=\zeta_{+}$and $\psi=\zeta_{-}$, it suffices to show that $\rho \psi=\zeta, \bar{\rho}=\rho$, and $\bar{\psi}=\psi^{-1}$.

Since $\rho$ vanishes on all compositions of $n$ when $n$ is odd, we have $\bar{\rho}=\rho$.

We show that $\rho \psi=\zeta$. Let $k_{e}:=k_{e}(\alpha)$ and $k_{o}:=k_{o}(\alpha)$.

Case 1. Suppose that $k=1$, so $\alpha=(n)$. We have $\rho\left(M_{(n)}\right)=1$ if $n$ is even and 0 if $n$ is odd; also $\psi\left(M_{(n)}\right)=0$ if $n$ is even and 1 if $n$ is odd. Thus $(\rho \psi)\left(M_{(n)}\right)=\rho\left(M_{(n)}\right)+\psi\left(M_{(n)}\right)=$ $1=\zeta\left(M_{(n)}\right)$.

In all remaining cases $k>1$ and $\zeta\left(M_{\alpha}\right)=0$ by (2).

Case 2. Suppose that $k>1$ and $a_{k}$ is even. By (3) we have

$$
(\rho \psi)\left(M_{\alpha}\right)=\sum_{i=0}^{k-1} \rho\left(M_{\alpha_{i}}\right) \psi\left(M_{\alpha^{i}}\right)+\rho\left(M_{\alpha}\right)=0
$$

by the second alternative of (7) applied to $\alpha^{i}$ and the third alternative of (86) applied to $\alpha$.

Case 3. Suppose that $k>1, a_{k}$ is odd, and $a_{1}$ is even. In this case $\rho\left(M_{\alpha_{i}}\right)=0$ for each $i>1$, so by (3)

$$
\begin{aligned}
& (\rho \psi)\left(M_{\alpha}\right)=\psi\left(M_{\alpha}\right)+\rho\left(M_{\left(a_{1}\right)}\right) \psi\left(M_{\left(a_{2}, \ldots, a_{k}\right)}\right)= \\
& \quad \frac{(-1)^{k_{e}}}{2^{2\left\lfloor k_{o} / 2\right\rfloor}} C\left(0,\left\lfloor k_{o} / 2\right\rfloor\right)+\frac{(-1)^{k_{e}-1}}{2^{2\left\lfloor k_{o} / 2\right\rfloor}} C\left(0,\left\lfloor k_{o} / 2\right\rfloor\right)=0 .
\end{aligned}
$$

Case 4. Suppose that $k>1$ and $a_{k}, a_{1}$, and $n$ are odd. By (8), we have $\rho\left(M_{\alpha_{i}}\right)=0$ unless $i=0$ or $a_{i}$ is odd and $\left|\alpha_{i}\right|$ is even. Hence

$$
\begin{aligned}
(-1)^{k_{e}} 2^{k_{o}-1}(\rho \psi)\left(M_{\alpha}\right) & =(-1)^{k_{e}} 2^{k_{o}-1}\left(\psi\left(M_{\alpha}\right)+\sum_{\substack{1 \leq i \leq k-1 \\
a_{i} \text { odd } \\
\left|\alpha_{i}\right| \text { even }}} \rho\left(M_{\alpha_{i}}\right) \psi\left(M_{\alpha^{i}}\right)\right) \\
& =B\left(\frac{k_{o}(\alpha)-1}{2}\right)-2 \sum_{\substack{1 \leq i \leq k-1 \\
a_{i} \text { odd } \\
\left|\alpha_{i}\right| \text { even }}} C\left(\frac{k_{o}\left(\alpha_{i}\right)}{2}-1\right) B\left(\frac{k_{o}\left(\alpha^{i}\right)-1}{2}\right) .
\end{aligned}
$$


We used (6) and the fact that $\lfloor h / 2\rfloor=\frac{h-1}{2}$ when $h$ is odd.

Deleting the even parts of $\alpha$ and changing every odd part of $\alpha$ to 1 does not change the right-hand side of this equation. Thus we may assume without loss of generality that $\alpha=(1,1, \ldots, 1) \vDash n=2 m+1$. In this case,

$$
(-1)^{k_{e}} 2^{k_{o}-1}(\rho \psi)\left(M_{\alpha}\right)=B(m)-2 \sum_{j=1}^{m} C(j-1) B(m-j)=0,
$$

by Lemma 3.1 .

Case 5. Suppose that $k>1, a_{k}$ and $a_{1}$ are odd, and $n$ is even. Then

$$
\begin{gathered}
(-1)^{k_{e}} 2^{k_{o}}(\rho \psi)\left(M_{\alpha}\right)=(-1)^{k_{e}} 2^{k_{o}}\left(\psi\left(M_{\alpha}\right)+\sum_{\substack{1 \leq i \leq k-1 \\
a_{i} \text { odd } \\
\left|\alpha_{i}\right| \text { even }}} \rho\left(M_{\alpha_{i}}\right) \psi\left(M_{\alpha^{i}}\right)+\rho\left(M_{\alpha}\right)\right) \\
=B\left(\frac{k_{o}(\alpha)}{2}\right)-2 \sum_{\substack{1 \leq i \leq k-1 \\
a_{i} \text { odd } \\
\left|\alpha_{i}\right| \text { even }}} C\left(\frac{k_{o}\left(\alpha_{i}\right)}{2}-1\right) B\left(\frac{k_{o}\left(\alpha^{i}\right)}{2}\right)-2 C\left(\frac{k_{o}(\alpha)}{2}-1\right) .
\end{gathered}
$$

As before, it suffices to consider the special case $\alpha=(1,1, \ldots, 1) \vDash 2 m$. In this case,

$$
\begin{aligned}
(-1)^{k_{e}} 2^{k_{o}}(\rho \psi)\left(M_{\alpha}\right) & =B(m)-2 \sum_{j=1}^{m-1} C(j-1) B(m-j)-2 C(m-1) \\
& =B(m)-2 \sum_{j=1}^{m} C(j-1) B(m-j)=0
\end{aligned}
$$

again by Lemma 3.1.

It remains to show that $\psi^{-1}=\bar{\psi}$. Since $\psi$ is invertible $(\psi(1)=1)$, it suffices to show that $\psi \bar{\psi}=\varepsilon$.

Case 1. If $a_{k}$ is even then $\bar{\psi}\left(M_{\alpha^{i}}\right)=0$ for every $i<k$ and $\psi\left(M_{\alpha}\right)=0$ by (77), so $(\psi \bar{\psi})\left(M_{\alpha}\right)=0$.

Case 2. Suppose $a_{k}$ and $n$ are odd. In this case $\bar{\psi}\left(M_{\alpha}\right)=-\psi\left(M_{\alpha}\right)$, so $(\psi \bar{\psi})\left(M_{\alpha}\right)=$ $\sum_{i=1}^{k-1} \psi\left(M_{\alpha_{i}}\right) \bar{\psi}\left(M_{\alpha^{i}}\right)$. By (6) $), k_{o}\left(\alpha_{i}\right)+k_{o}\left(\alpha^{i}\right)=k_{o}(\alpha)$ is odd. Hence

$$
\left\lfloor k_{o}\left(\alpha_{i}\right) / 2\right\rfloor+\left\lfloor k_{o}\left(\alpha^{i}\right) / 2\right\rfloor=\frac{k_{o}(\alpha)-1}{2} \text { and } k_{e}\left(\alpha_{i}\right)+k_{e}\left(\alpha^{i}\right)+\left|\alpha^{i}\right| \equiv k(\alpha)+\left|\alpha_{i}\right| \bmod 2 .
$$

It follows that

$$
(-1)^{k(\alpha)} 2^{k_{o}(\alpha)-1}(\psi \bar{\psi})\left(M_{\alpha}\right)=\sum_{\substack{1 \leq i \leq k-1 \\ a_{i} \text { odd }}}(-1)^{\left|\alpha_{i}\right|} B\left(\left\lfloor k_{o}\left(\alpha_{i}\right) / 2\right\rfloor\right) B\left(\left\lfloor k_{o}\left(\alpha^{i}\right) / 2\right\rfloor\right) .
$$


As before, we may assume $\alpha=(1,1, \ldots, 1) \vDash n=2 m+1$, in which case the above sum becomes

$$
\sum_{i=1}^{2 m}(-1)^{i} B(\lfloor i / 2\rfloor) B(\lfloor(2 m+1-i) / 2\rfloor) .
$$

As $j$ runs from 1 to $m$, the terms in this sum corresponding to $i=2 j$ and $i=2 m-2 j+1$ are $B(j) B(m-j)$ and $-B(m-j) B(j)$, respectively. Since this covers all terms, this sum is zero.

Case 3. Suppose $a_{k}$ is odd and $n$ is even. Similar considerations lead to

$$
2 B\left(\frac{k_{o}(\alpha)}{2}\right)+\sum_{\substack{1 \leq i \leq k-1 \\ a_{i} \text { odd } \\\left|\alpha_{i}\right| \text { even }}} B\left(\frac{k_{o}\left(\alpha_{i}\right)}{2}\right) B\left(\frac{k_{o}\left(\alpha^{i}\right)}{2}\right)-2^{2} \sum_{\substack{1 \leq i \leq k-1 \\ a_{i} \text { odd } \\\left|\alpha_{i}\right| \text { odd }}} B\left(\frac{k_{o}\left(\alpha_{i}\right)-1}{2}\right) B\left(\frac{k_{o}\left(\alpha^{i}\right)-1}{2}\right) .
$$

Once again, we may assume $\alpha=(1,1, \ldots, 1) \vDash n=2 m$. Then showing that $(\psi \bar{\psi})\left(M_{\alpha}\right)=0$ is equivalent to showing that

$$
\sum_{i=0}^{m} B(i) B(m-i)=2^{2} \sum_{i=0}^{m-1} B(i) B(m-1-i) .
$$

This equality follows from (5) in Lemma 3.1 .

The proof is complete.

\section{Application: Identities for Catalan numbers and central binomial coefficients}

In the proof of Theorem [3.2, we did not need to show that the functionals defined by (77), (8) are characters (morphisms of algebras); indeed, this fact follows from our argument. We may derive interesting identities involving Catalan numbers or central binomial coefficients from this property. To this end, we first describe the product of two monomial quasi-symmetric functions. This result is known from [8, Lemma 3.3], [15, [16], and [24, Section 5]. We present here an equivalent but more convenient description due to Fares [10].

Given non-negative integers $p$ and $q$, consider the set $\mathcal{L}(p, q)$ of lattice paths from $(0,0)$ to $(p, q)$ consisting of unit steps which are either horizontal, vertical, or diagonal (usually called Delannoy paths). An element of $\mathcal{L}(p, q)$ is thus a sequence $L=\left(\ell_{1}, \ldots, \ell_{s}\right)$ such that each $\ell_{i}$ is either $(1,0),(0,1)$, or $(1,1)$, and $\sum \ell_{i}=(p, q)$. Let $h, v$, and $d$ be the number of horizontal, vertical, and diagonal steps in $L$. Then $h+d=p, v+d=q$, and $s=h+v+d=p+q-d$. The number of lattice paths in $\mathcal{L}(p, q)$ with $d$ diagonal steps is the multinomial coefficient

$$
\left(\begin{array}{c}
s \\
h, v, d
\end{array}\right)=\left(\begin{array}{c}
p+q-d \\
p-d, q-d, d
\end{array}\right)
$$


since such a path is determined by the decomposition of the set of steps into the subsets of horizontal, vertical, and diagonal steps.

Given compositions $\alpha=\left(a_{1}, \ldots, a_{p}\right)$ and $\beta=\left(b_{1}, \ldots, b_{q}\right)$ and $L \in \mathcal{L}(p, q)$, we label each step of $L$ according to its horizontal and vertical projections, as indicated in the example below $(p=5, q=4)$ :
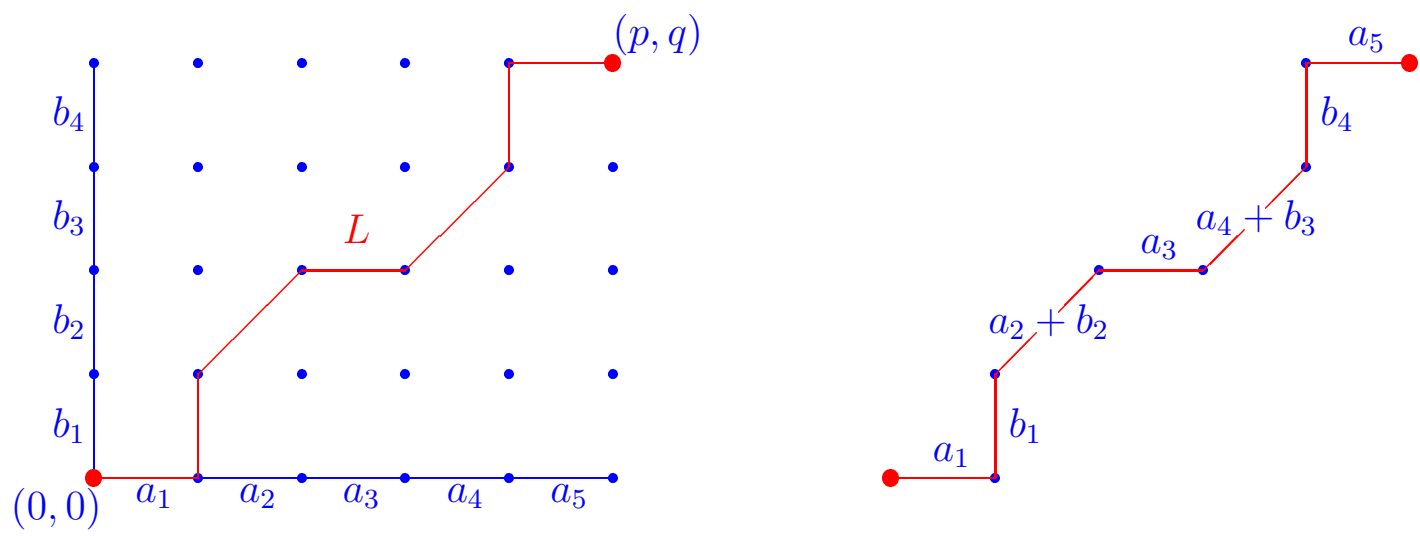

Then we obtain a composition $q_{L}(\alpha, \beta)$ by reading off the labels along the path $L$ in order. In the example above,

$$
q_{L}(\alpha, \beta)=\left(a_{1}, b_{1}, a_{2}+b_{2}, a_{3}, a_{4}+b_{3}, b_{4}, a_{5}\right) .
$$

The composition $q_{L}(\alpha, \beta)$ is the quasi-shuffle of $\alpha$ and $\beta$ corresponding to $L$. If $L$ does not involve diagonal steps, then $q_{L}(\alpha, \beta)$ is an ordinary shuffle.

The product of two monomial quasi-symmetric functions is given by

$$
M_{\alpha} \cdot M_{\beta}=\sum_{L \in \mathcal{L}(p, q)} M_{q_{L}(\alpha, \beta)} .
$$

For our first application we make use of the fact that $\zeta_{-}$is a character.

Corollary 4.1. Let $n, m$ be non-negative integers not both equal to 0 . Then

$$
\begin{aligned}
& \sum_{d=0}^{\min (n, m)} \frac{(-1)^{d}}{4^{\lfloor(n+m-2 d) / 2\rfloor}} \frac{n+m-2 d}{n+m-d}\left(\begin{array}{c}
n+m-d \\
n-d, m-d, d
\end{array}\right)\left(\begin{array}{c}
2\lfloor(n+m-2 d) / 2\rfloor \\
\lfloor(n+m-2 d) / 2\rfloor
\end{array}\right) \\
& =\frac{1}{4^{\lfloor n / 2\rfloor+\lfloor m / 2\rfloor}}\left(\begin{array}{c}
2\lfloor n / 2\rfloor \\
\lfloor n / 2\rfloor
\end{array}\right)\left(\begin{array}{c}
2\lfloor m / 2\rfloor \\
\lfloor m / 2\rfloor
\end{array}\right) .
\end{aligned}
$$

Proof. Let $\alpha=(1,1, \ldots, 1) \vDash n$ and $\beta=(1,1, \ldots, 1) \vDash m$. The set of lattice paths $\mathcal{L}(n, m)$ splits as

$$
\mathcal{L}(n, m)=\mathcal{L}_{H}(n, m) \sqcup \mathcal{L}_{V}(n, m) \sqcup \mathcal{L}_{D}(n, m)
$$

according to whether the last step of the path is horizontal, vertical, or diagonal.

Choose $L \in \mathcal{L}(n, m)$, let $d$ be the number of diagonal steps of $L$, and $\gamma:=q_{L}(\alpha, \beta)$. 
If $L \in \mathcal{L}_{D}(n, m)$, the last part of $\gamma$ is 2 , so $\zeta_{-}\left(M_{\gamma}\right)=0$ by (7). On the other hand, if $L \in \mathcal{L}_{H}(n, m) \sqcup \mathcal{L}_{V}(n, m)$ the last part of $\gamma$ is $1, k_{e}(\gamma)=d$ ( $d$ parts are equal to 2$)$, and $k_{o}(\gamma)=n+m-2 d$ (all other parts are equal to 1$)$. Hence, by (7),

$$
\zeta_{-}\left(M_{\gamma}\right)=\frac{(-1)^{d}}{2^{2\lfloor(n+m-2 d) / 2\rfloor}} C(0,\lfloor(n+m-2 d) / 2\rfloor)
$$

Now, the number of paths in $\mathcal{L}_{H}(n, m) \sqcup \mathcal{L}_{V}(n, m)$ with $d$ diagonal steps is

$$
\left(\begin{array}{c}
n-1+m-d \\
n-1-d, m-d, d
\end{array}\right)+\left(\begin{array}{c}
n+m-1-d \\
n-d, m-1-d, d
\end{array}\right)=\frac{n+m-2 d}{n+m-d}\left(\begin{array}{c}
n+m-d \\
n-d, m-d, d
\end{array}\right) .
$$

Therefore, by (9),

$$
\zeta_{-}\left(M_{\alpha} \cdot M_{\beta}\right)=\sum_{d=0}^{\min (n, m)} \frac{(-1)^{d}}{2^{2\lfloor(n+m-2 d) / 2\rfloor}} \frac{n+m-2 d}{n+m-d}\left(\begin{array}{c}
n+m-d \\
n-d, m-d, d
\end{array}\right) C(0,\lfloor(n+m-2 d) / 2\rfloor) .
$$

On the other hand,

$$
\zeta_{-}\left(M_{\alpha}\right) \cdot \zeta_{-}\left(M_{\beta}\right)=\frac{1}{2^{2\lfloor n / 2\rfloor}} C(0,\lfloor n / 2\rfloor) \cdot \frac{1}{2^{2\lfloor m / 2\rfloor}} C(0,\lfloor m / 2\rfloor) .
$$

Since $\zeta_{-}$is a character, the last two quantities are equal. Equating them results in (10).

The special case of (10) when $n \geq m=1$ is

$$
\frac{n+1}{4^{\lfloor(n+1) / 2\rfloor}}\left(\begin{array}{c}
2\lfloor(n+1) / 2\rfloor \\
\lfloor(n+1) / 2\rfloor
\end{array}\right)-\frac{n-1}{4^{\lfloor(n-1) / 2\rfloor}}\left(\begin{array}{c}
2\lfloor(n-1) / 2\rfloor \\
\lfloor(n-1) / 2\rfloor
\end{array}\right)=\frac{1}{4^{\lfloor n / 2\rfloor}}\left(\begin{array}{c}
2\lfloor n / 2\rfloor \\
\lfloor n / 2\rfloor
\end{array}\right)
$$

which may be easily verified. On the other hand, when $n=m \geq 1$, (10) becomes

$$
\sum_{d=0}^{n-1} \frac{(-1)^{d}}{4^{n-d}}\left(\begin{array}{c}
2 n-d-1 \\
d
\end{array}\right)\left(\begin{array}{c}
2(n-d) \\
n-d
\end{array}\right)^{2}=\frac{1}{4^{2\lfloor n / 2\rfloor}}\left(\begin{array}{c}
2\lfloor n / 2\rfloor \\
\lfloor n / 2\rfloor
\end{array}\right)^{2}
$$

We now make use of the fact that $\zeta_{+}$is a character to derive an identity involving Catalan numbers.

Corollary 4.2. Let $n, m$ be positive integers not both equal to 1 and such that $n \equiv m$ $\bmod 2$. Then

$$
\begin{array}{r}
\sum_{d=0}^{\min (n, m)}(-1)^{d+1} 2^{2 d-1} \frac{(n+m-2 d)}{(n+m-d)} \frac{(n+m-2 d-1)}{(n+m-d-1)}\left(\begin{array}{c}
n+m-d \\
n-d, m-d, d
\end{array}\right) C((n+m) / 2-d-1) \\
= \begin{cases}C(n / 2-1) C(m / 2-1) & \text { if } n \text { and } m \text { are even, } \\
0 & \text { if } n \text { and } m \text { are odd. }\end{cases}
\end{array}
$$


Proof. Let $\alpha=(1,1, \ldots, 1) \vDash n$ and $\beta=(1,1, \ldots, 1) \vDash m$. As in the proof of Corollary 4.1, we analyze which lattice paths $L \in \mathcal{L}(n, m)$ contribute to $\zeta_{+}\left(M_{\alpha} \cdot M_{\beta}\right)$. Note that since $(n, m) \neq(1,1)$, the second alternative in (8) never occurs. A path which starts or ends with a diagonal step does not contribute, by the third alternative in (8). For all remaining paths $L$, if $d$ is the number of diagonal steps, then

$$
\zeta_{+}\left(M_{q_{L}(\alpha, \beta)}\right)=\frac{(-1)^{d+1}}{2^{n+m-2 d-1}} C((n+m-2 d) / 2-1)
$$

by the first alternative in (8). These paths start and end with a horizontal or vertical step, so their number is

$$
\begin{aligned}
2\left(\begin{array}{c}
n-1+m-1-d \\
n-1-d, m-1-d, d
\end{array}\right)+ & \left(\begin{array}{c}
n-2+m-d \\
n-2-d, m-d, d
\end{array}\right)+\left(\begin{array}{c}
n+m-2-d \\
n-d, m-2-d, d
\end{array}\right) \\
& =\frac{(n+m-2 d)}{(n+m-d)} \frac{(n+m-2 d-1)}{(n+m-d-1)}\left(\begin{array}{c}
n+m-d \\
n-d, m-d, d
\end{array}\right) .
\end{aligned}
$$

Applying $\zeta_{+}$to both sides of (9) leads to (11).

Suppose $m=1, n=2 k+1, k \geq 1$. In this case (11) boils down to the simple identity

$$
C(k)=\frac{2(2 k-1)}{k+1} C(k-1) .
$$

If $n=m$, the last term in the sum (11) is 0 , because of the factor $n+m-2 d$ (so there is no need to evaluate the Catalan number in this case). The formula becomes

$$
\sum_{d=0}^{n-1}(-1)^{d+1} 4^{d}(2 n-2 d-1)\left(\begin{array}{c}
2 n-d-2 \\
d
\end{array}\right) C(n-d-1)^{2}= \begin{cases}C(n / 2-1)^{2} & \text { if } n \text { is even, } \\
0 & \text { if } n \text { is odd, } n>1 .\end{cases}
$$

In the proof of Theorem 3.2 we established that formula (7) defines an odd character by showing that $\zeta_{-} \bar{\zeta}_{-}=\epsilon$. Rewriting this property in terms of the antipode $S$ of $\mathcal{Q} S y m$ leads to new combinatorial identities that we analyze next. First, recall that $S$ is given by

$$
S\left(M_{\beta}\right)=(-1)^{k(\beta)} \sum_{\alpha \leq \tilde{\beta}} M_{\alpha}
$$

where $\tilde{\beta}=\left(b_{k}, \ldots, b_{2}, b_{1}\right)$ is the reversal of $\beta=\left(b_{1}, b_{2}, \ldots, b_{k}\right)$ and $\alpha \leq \gamma$ indicates that $\gamma$ is a refinement of $\alpha$ [8, Proposition 3.4]; [17, Corollaire 4.20].

Corollary 4.3. For any composition $\beta=\left(b_{1}, \ldots, b_{k}\right)$,

$$
\sum_{\substack{\alpha \leq \beta \\
a_{1} \text { odd }}} \frac{(-1)^{k_{e}(\alpha)}}{4^{\left\lfloor k_{o}(\alpha) / 2\right\rfloor}}\left(\begin{array}{c}
2\left\lfloor k_{o}(\alpha) / 2\right\rfloor \\
\left.k_{o}(\alpha) / 2\right\rfloor
\end{array}\right)= \begin{cases}\frac{1}{4^{\left\lfloor k_{o}(\beta) / 2\right\rfloor}}\left(\begin{array}{c}
2\left\lfloor k_{o}(\beta) / 2\right\rfloor \\
\left.k_{o}(\beta) / 2\right\rfloor
\end{array}\right) & \text { if } b_{k} \text { is odd } \\
0 & \text { if } b_{k} \text { is even }\end{cases}
$$

the sum being over those compositions $\alpha=\left(a_{1}, \ldots, a_{h}\right)$ whose first part is odd and which are refined by $\beta$. 
Proof. Since $\zeta_{-}$is an odd character, we have

$$
\zeta_{-} \circ S=\left(\zeta_{-}\right)^{-1}=\bar{\zeta}_{-} .
$$

Therefore, for any composition $\beta$ of $n$ we have, by (12),

$$
(-1)^{k(\beta)} \sum_{\alpha \leq \tilde{\beta}} \zeta_{-}\left(M_{\alpha}\right)=(-1)^{n} \zeta_{-}\left(M_{\beta}\right) .
$$

Hence, by (7),

$$
(-1)^{k(\beta)} \sum_{\substack{\alpha \leq \tilde{\beta} \\ a_{h} \text { odd }}} \frac{(-1)^{k_{e}(\alpha)}}{2^{2\left\lfloor k_{o}(\alpha) / 2\right\rfloor}} C\left(0,\left\lfloor k_{o}(\alpha) / 2\right\rfloor\right)= \begin{cases}\frac{(-1)^{n+k_{e}(\beta)}}{2^{2\left\lfloor k_{o}(\beta) / 2\right\rfloor}} C\left(0,\left\lfloor k_{o}(\beta) / 2\right\rfloor\right) & \text { if } b_{k} \text { is odd, } \\ 0 & \text { if } b_{k} \text { is even. }\end{cases}
$$

By (㧫), $k(\beta)=k_{o}(\beta)+k_{e}(\beta) \equiv n+k_{e}(\beta)$. This is equivalent to (13).

Suppose that $k_{e}(\beta) \equiv 0 \bmod 2$ and $b_{1} \equiv b_{k} \bmod 2$. In this case, the summand in (13) corresponding to $\alpha=\beta$ cancels with the right-hand side. We deduce that for any such composition $\beta$,

$$
\sum_{\substack{\alpha<\beta \\
a_{1} \text { odd }}} \frac{(-1)^{k_{e}(\alpha)}}{4^{\left\lfloor k_{o}(\alpha) / 2\right\rfloor}}\left(\begin{array}{c}
2\left\lfloor k_{o}(\alpha) / 2\right\rfloor \\
\left\lfloor k_{o}(\alpha) / 2\right\rfloor
\end{array}\right)=0 .
$$

\section{The canonical characters of $\mathcal{Q} S y m$ on the funda- mental basis}

For a composition $\alpha=\left(a_{1}, \ldots, a_{k}\right)$ of a non-negative integer $n$, define

$$
\begin{aligned}
& p_{-}(\alpha)=\#\left\{i \neq k: a_{i}>1\right\}, \\
& p_{+}(\alpha)= \begin{cases}1+\#\left\{i \neq 1, k: a_{i}>1\right\} & \text { if } k>1, \\
0 & \text { if } k \leq 1 .\end{cases}
\end{aligned}
$$

A combinatorial interpretation for these two statistics in terms of peaks of permutations is given in Section 7

A composition $\alpha=\left(a_{1}, \ldots, a_{k}\right)$ may be conveniently represented by a ribbon diagram: a sequence of rows of squares, each row consisting of $a_{i}$ squares, and with the first square in row $i+1$ directly below the last square in row $i$. For instance the diagram

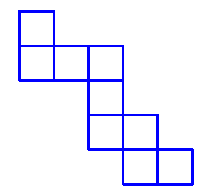

represents the composition $(1,3,1,2,2)$. Note that $p_{-}(\alpha)$ is the number of upper corners in the ribbon diagram of $\alpha$. To get a similar interpretation for $p_{+}(\alpha)$ one may augment the 
ribbon diagram of $\alpha$ by drawing an extra square to the left of the first row. Then $p_{+}(\alpha)$ is the number of upper corners in the augmented diagram. For instance, $p_{-}(1,3,1,2,2)=2$ and $p_{+}(1,3,1,2,2)=3$, as illustrated below.
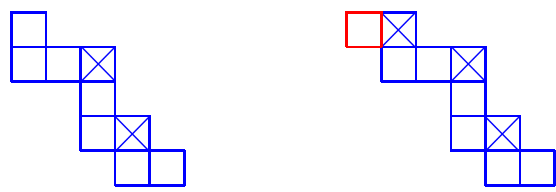

The fundamental and monomial bases of $\mathcal{Q} S y m$ are related by

$$
M_{\alpha}=\sum_{\beta \geq \alpha}(-1)^{k(\beta)-k(\alpha)} F_{\beta}
$$

where, as before, for compositions $\alpha$ and $\beta$ of $n, \beta \geq \alpha$ indicates that $\beta$ is refinement of $\alpha$.

Theorem 5.1. Let $\alpha$ be a composition of a non-negative integer $n$. Then

$$
\begin{aligned}
& \zeta_{-}\left(F_{\alpha}\right)=\frac{(-1)^{p_{-}(\alpha)}}{2^{2\lfloor n / 2\rfloor} C\left(p_{-}(\alpha),\lfloor n / 2\rfloor-p_{-}(\alpha)\right) ;} \\
& \zeta_{+}\left(F_{\alpha}\right)= \begin{cases}\frac{(-1)^{p_{+}(\alpha)}}{2^{n}} C\left(p_{+}(\alpha), n / 2-p_{+}(\alpha)\right) & \text { if } n \text { is even, } \\
0 & \text { if } n \text { is odd. }\end{cases}
\end{aligned}
$$

Remark 5.2. Up to a sign and a factor of $\pi$, the above are values of Legendre's beta function at half-integers. Indeed, as already remarked by Catalan [4, p. 15], [6, Section $\mathrm{CV}]$,

$$
\frac{1}{2^{2(p+q)}} C(p, q)=\frac{1}{\pi} B\left(p+\frac{1}{2}, q+\frac{1}{2}\right)=\frac{1}{\pi} \int_{0}^{\pi} \sin ^{2 p} \theta \cos ^{2 q} \theta d \theta=\frac{1}{\pi} \int_{0}^{1} \frac{(1-u)^{p} u^{q}}{\sqrt{(1-u) u}} d u
$$

This specializes to an integral representation for the Catalan numbers, equivalent to the one given in [18] and [22, Problem 6.C13].

Remark 5.3. Catalan shows [6, Section CCXIV], 7, pp. 110-113] that $C(p, q)$ is an integer, and that the highest power of 2 that divides it equals the number of 1 's in the binary decomposition of $p+q$. Equivalently, the above fraction may be reduced as follows

$$
\frac{1}{2^{2(p+q)}} C(p, q)=\frac{N}{2^{k}} \text { with } N \text { odd and } k=\sum_{i \geq 0}\left\lfloor\frac{p+q}{2^{i}}\right\rfloor .
$$

The proof of Theorem 5.1 is given below. We need two more statistics. For $\alpha$ as above, let

$$
u(\alpha)=\#\left\{i \neq 1: a_{i}>1\right\} \text { and } v(\alpha)=\#\left\{i: a_{i}>1\right\}
$$


Lemma 5.4. Let $m, j$ be non-negative integers. Then
(a) $\sum_{\substack{\gamma \vDash m \\ v(\gamma)=j}}(-1)^{k(\gamma)}=(-1)^{m+j}\left(\begin{array}{c}\lfloor m / 2\rfloor \\ j\end{array}\right)$;
(b) $\sum_{\substack{\gamma \vDash m \\ u(\gamma)=j}}(-1)^{k(\gamma)}= \begin{cases}0 & \text { if } m \text { is even, } \\ (-1)^{m+j}\left(\begin{array}{c}\lfloor m / 2\rfloor \\ j\end{array}\right) & \text { if } m \text { is odd. }\end{cases}$

We thank Ira Gessel for supplying this proof.

Proof. The generating function for the left-hand side of (a) multiplied by $y^{j} x^{m}$ is just the sum of weights of all compositions, where a part $a>1$ is weighted by $-y x^{a}$ and a part equal to 1 is weighted by $-x$. This sum is

$$
\frac{1}{1-\left(-x-y x^{2}-y x^{3}+\cdots\right)}=\frac{1}{1+x+\frac{y x^{2}}{1-x}}=\frac{1-x}{1-(1-y) x^{2}} .
$$

By expanding in powers of $x$ and $y$ we obtain the identity (a). In the generating function for $(\mathrm{b})$, the first part $a$ is weighted $-x^{a}$ no matter what $a$ is, so this function is $-x /(1-x)$ times the generating function for $(\mathrm{a})$. This is $-x /\left(1-(1-y) x^{2}\right)$, which is the odd powers of $x$ in (a).

Lemma 5.5. Let $i, j, m$ be non-negative integers. Then

$$
2^{2 m} C(i, j)=\sum_{b=0}^{m}\left(\begin{array}{c}
m \\
b
\end{array}\right) C(i+b, m+j-b) .
$$

Proof. In view of (20), the proposed equality is equivalent to

$$
\int_{0}^{\pi} \sin ^{2 i} \theta \cos ^{2 j} \theta d \theta=\sum_{b=0}^{m}\left(\begin{array}{c}
m \\
b
\end{array}\right) \int_{0}^{\pi} \sin ^{2(i+b)} \theta \cos ^{2(m+j-b)} \theta d \theta .
$$

This holds since

$$
\sum_{b=0}^{m}\left(\begin{array}{c}
m \\
b
\end{array}\right) \sin ^{2 b} \theta \cos ^{2(m-b)} \theta=\left(\sin ^{2} \theta+\cos ^{2} \theta\right)^{m}=1 .
$$

To facilitate the proof of Theorem 5.1 we define

$$
\begin{aligned}
& H_{-}(\alpha)=\sum_{\beta \geq \alpha}(-1)^{k(\beta)+p_{-}(\beta)+1} C\left(p_{-}(\beta),\lfloor n / 2\rfloor-p_{-}(\beta)\right) \quad \text { for } \alpha \vDash n ; \\
& H_{+}(\alpha)=\sum_{\beta \geq \alpha}(-1)^{k(\beta)+p_{+}(\beta)+1} C\left(p_{+}(\beta), n / 2-p_{+}(\beta)\right) \quad \text { for } \alpha \vDash n, n \text { even. }
\end{aligned}
$$


Lemma 5.6. Suppose that $\alpha=\left(a_{1}, \ldots, a_{k}\right) \vDash n$. Then

$$
H_{-}(\alpha)= \begin{cases}(-1)^{n-1} 2^{n-k_{o}(\alpha)} C\left(0,\left\lfloor k_{o}(\alpha) / 2\right\rfloor\right) & \text { if } a_{k} \text { is odd, } \\ 0 & \text { if } a_{k} \text { is even. }\end{cases}
$$

Proof. We will consider the cases $\alpha=(n)$ and $\alpha \neq(n)$ separately.

Case 1. Suppose that $\alpha=(n)$. We need to show that $H_{-}((n))=2^{n-1}$ if $n$ is odd and $H_{-}((n))=0$ if $n$ is even. For a composition $\gamma$ and a positive integer $i$, let $\gamma i$ denote the concatenation of $\gamma$ with $(i)$. Using the fact that $p_{-}(\gamma i)=v(\gamma)$, we have

$$
\begin{aligned}
H_{-}((n)) & =\sum_{\beta \models n}(-1)^{k(\beta)+p_{-}(\beta)+1} C\left(p_{-}(\beta),\lfloor n / 2\rfloor-p_{-}(\beta)\right) \\
& =C(0,\lfloor n / 2\rfloor)+\sum_{i=1}^{n-1} \sum_{\gamma \models n-i}(-1)^{k(\gamma i)+p_{-}(\gamma i)+1} C\left(p_{-}(\gamma i),\lfloor n / 2\rfloor-p_{-}(\gamma i)\right) \\
& =C(0,\lfloor n / 2\rfloor)+\sum_{i=1}^{n-1} \sum_{\gamma \neq n-i}(-1)^{k(\gamma)+v(\gamma)} C(v(\gamma),\lfloor n / 2\rfloor-v(\gamma)) \\
& =C(0,\lfloor n / 2\rfloor)+\sum_{i=1}^{n-1} \sum_{b=0}^{\lfloor(n-i) / 2\rfloor} \sum_{\substack{\gamma \neq n-i \\
v(\gamma)=b}}(-1)^{k(\gamma)+b} C(b,\lfloor n / 2\rfloor-b) \\
& =C(0,\lfloor n / 2\rfloor)+\sum_{i=1}^{n-1} \sum_{b=0}^{\lfloor(n-i) / 2\rfloor}(-1)^{b} C(b,\lfloor n / 2\rfloor-b) \sum_{\substack{\gamma \in n-i \\
v(\gamma)=b}}(-1)^{k(\gamma)} .
\end{aligned}
$$

Make the substitution $m=n-i$ and apply Lemma 5.4(a) to get

$$
\begin{aligned}
H_{-}((n)) & =C(0,\lfloor n / 2\rfloor)+\sum_{m=1}^{n-1} \sum_{b=0}^{\lfloor m / 2\rfloor}(-1)^{m}\left(\begin{array}{c}
\lfloor m / 2\rfloor \\
b
\end{array}\right) C(b,\lfloor n / 2\rfloor-b) \\
& =C(0,\lfloor n / 2\rfloor)+\sum_{b=0}^{\lfloor n-1 / 2\rfloor} C(b,\lfloor n / 2\rfloor-b) \sum_{m=1}^{n-1}(-1)^{m}\left(\begin{array}{c}
\lfloor m / 2\rfloor \\
b
\end{array}\right) .
\end{aligned}
$$

Let $S_{b}=\sum_{m=1}^{n-1}(-1)^{m}\left(\begin{array}{c}\lfloor m / 2\rfloor \\ b\end{array}\right)$. If $m$ is even, $(-1)^{m}\left(\begin{array}{c}\lfloor m / 2\rfloor \\ b\end{array}\right)+(-1)^{m+1}\left(\begin{array}{c}\lfloor(m+1) / 2\rfloor \\ b\end{array}\right)=0$. Hence all terms in the sum $S_{b}$ cancel, except possibly for the first and the last.

If $n$ is even, then $S_{b}=0$ when $b \geq 1$ and $S_{0}=-1$; hence $H_{-}((n))=C(0,\lfloor n / 2\rfloor)+$ $[-C(0,\lfloor n / 2\rfloor)]=0$. If $n$ is odd, then $S_{b}=\left(\begin{array}{c}\lfloor n / 2\rfloor \\ b\end{array}\right)$ when $b \geq 1$ and $S_{0}=0$; hence by Lemma 5.5 (with $i=j=0$ ),

$$
\begin{aligned}
H_{-}((n)) & =C(0,\lfloor n / 2\rfloor)+\sum_{b=1}^{\lfloor n / 2\rfloor} C(b,\lfloor n / 2\rfloor-b)\left(\begin{array}{c}
\lfloor n / 2\rfloor \\
b
\end{array}\right) \\
& =\sum_{b=0}^{\lfloor n / 2\rfloor}\left(\begin{array}{c}
\lfloor n / 2\rfloor \\
b
\end{array}\right) C(b,\lfloor n / 2\rfloor-b) \\
& =2^{2\lfloor n / 2\rfloor} .
\end{aligned}
$$


Moreover, we have $2\lfloor n / 2\rfloor=n-1$ since $n$ is odd.

Case 2. Suppose that $\alpha \neq(n)$. If $\beta$ and $\gamma$ are non-empty compositions and $\beta \gamma$ denotes their concatenation, then $p_{-}(\beta \gamma)=v(\beta)+u(\tilde{\gamma})$, where $\tilde{\gamma}$ is the reversal of $\gamma$. Using this observation we may write

$$
\begin{aligned}
H_{-}(\alpha) & =\sum_{\substack{\beta \geq \alpha_{k-1} \\
\gamma \vDash a_{k}}}(-1)^{k(\beta \gamma)+p_{-}(\beta \gamma)} C\left(p_{-}(\beta \gamma),\lfloor n / 2\rfloor-p_{-}(\beta \gamma)\right) \\
& =\sum_{\beta \geq \alpha_{k-1}}(-1)^{k(\beta)+v(\beta)} \sum_{\substack{\gamma \vDash a_{k}\\
}}(-1)^{k(\gamma)+u(\tilde{\gamma})} C(v(\beta)+u(\tilde{\gamma}),\lfloor n / 2\rfloor-v(\beta)-u(\tilde{\gamma})) \\
& =\sum_{\beta \geq \alpha_{k-1}}(-1)^{k(\beta)+v(\beta)} \sum_{b=0}^{\left\lfloor a_{k} / 2\right\rfloor}(-1)^{b} C(v(\beta)+b,\lfloor n / 2\rfloor-v(\beta)-b) \sum_{\substack{\gamma \neq a_{k} \\
u(\tilde{\gamma})=b}}(-1)^{k(\gamma)} .
\end{aligned}
$$

By Lemma 5.4(b), the second sum in (22) equals

$$
\begin{aligned}
& \begin{cases}(-1)^{a_{k}} \sum_{b=0}^{\left\lfloor a_{k} / 2\right\rfloor}\left(\begin{array}{c}
\left\lfloor a_{k} / 2\right\rfloor \\
b
\end{array}\right) C(v(\beta)+b,\lfloor n / 2\rfloor-v(\beta)-b) & \text { if } a_{k} \text { is odd, } \\
0 & \text { if } a_{k} \text { is even }\end{cases} \\
& = \begin{cases}(-1)^{a_{k}} 2^{2\left\lfloor a_{k} / 2\right\rfloor} C\left(v(\beta),\lfloor n / 2\rfloor-v(\beta)-\left\lfloor a_{k} / 2\right\rfloor\right) & \text { if } a_{k} \text { is odd, } \\
0 & \text { if } a_{k} \text { is even. }\end{cases}
\end{aligned}
$$

The last step uses Lemma 5.5. It follows that $H_{-}(\alpha)=0$ if $a_{k}$ is even.

Assume from now on that $a_{k}$ is odd. For any composition $\beta=\left(b_{1}, \ldots, b_{\ell}\right)$, let $\lfloor\beta / 2\rfloor=$ $\left\lfloor b_{1} / 2\right\rfloor+\cdots+\left\lfloor b_{\ell} / 2\right\rfloor$. We will show that for $0 \leq i<k$,

$$
H_{-}(\alpha)=(-1)^{\left|\alpha^{i}\right|} 2^{2\left\lfloor\alpha^{i} / 2\right\rfloor} \sum_{\beta \geq \alpha_{i}}(-1)^{k(\beta)+v(\beta)} C\left(v(\beta),\lfloor n / 2\rfloor-\left\lfloor\alpha^{i} / 2\right\rfloor-v(\beta)\right) .
$$

This holds for $i=k-1$, as can be seen by substituting the first alternative of (23) into the second sum in (22). Suppose by induction that (24) is true for some $i$ such that $k-1 \geq i \geq 1$. Since $k(\beta \gamma)=k(\beta)+k(\gamma)$ and $v(\beta \gamma)=v(\beta)+v(\gamma)$, we may rewrite (24) 
as

$$
\begin{aligned}
& H_{-}(\alpha)=(-1)^{\left|\alpha^{i}\right|} 2^{2\left\lfloor\alpha^{i} / 2\right\rfloor} \sum_{\substack{\delta \geq \alpha_{i-1} \\
\gamma \neq a_{i}}}(-1)^{k(\delta \gamma)+v(\delta \gamma)} C\left(v(\delta \gamma),\lfloor n / 2\rfloor-\left\lfloor\alpha^{i} / 2\right\rfloor-v(\delta \gamma)\right) \\
& =(-1)^{\left|\alpha^{i}\right|} 2^{2\left\lfloor\alpha^{i} / 2\right\rfloor} \sum_{\delta \geq \alpha_{i-1}}(-1)^{k(\delta)+v(\delta)} \sum_{\gamma \vDash a_{i}}(-1)^{k(\gamma)+v(\gamma)} \\
& C\left(v(\delta)+v(\gamma),\lfloor n / 2\rfloor-\left\lfloor\alpha^{i} / 2\right\rfloor-v(\delta)-v(\gamma)\right) \\
& =(-1)^{\left|\alpha^{i}\right|} 2^{2\left\lfloor\alpha^{i} / 2\right\rfloor} \sum_{\delta \geq \alpha_{i-1}}(-1)^{k(\delta)+v(\delta)} \sum_{b=0}^{\left\lfloor a_{i} / 2\right\rfloor}(-1)^{b} \\
& C\left(v(\delta)+b,\lfloor n / 2\rfloor-\left\lfloor\alpha^{i} / 2\right\rfloor-v(\delta)-b\right) \sum_{\substack{\gamma \models a_{i} \\
v(\gamma)=b}}(-1)^{k(\gamma)} \\
& =(-1)^{\left|\alpha^{i-1}\right|} 2^{2\left\lfloor\alpha^{i} / 2\right\rfloor} \sum_{\delta \geq \alpha_{i-1}}(-1)^{k(\delta)+v(\delta)} \\
& \sum_{b=0}^{\left\lfloor a_{i} / 2\right\rfloor} C\left(v(\delta)+b, n / 2-\left\lfloor\alpha^{i} / 2\right\rfloor-v(\delta)-b\right)\left(\begin{array}{c}
\left\lfloor a_{i} / 2\right\rfloor \\
b
\end{array}\right) .
\end{aligned}
$$

The last step uses Lemma 5.4(a). Now apply Lemma 5.5 with $i=v(\delta)$ and $j=\lfloor n / 2\rfloor-$ $\left\lfloor\alpha^{i-1} / 2\right\rfloor-v(\delta)$, to simplify the second sum in (25). The resulting formula is

$$
H_{-}(\alpha)=(-1)^{\left|\alpha^{i-1}\right|} 2^{2\left\lfloor\alpha^{i-1} / 2\right\rfloor} \sum_{\delta \geq \alpha_{i-1}}(-1)^{k(\delta)+v(\delta)} C\left(v(\delta),\lfloor n / 2\rfloor-\left\lfloor\alpha^{i-1} / 2\right\rfloor-v(\delta)\right) .
$$

This completes the proof of (24). Setting $i=0$ in (24) gives the formula stated in the lemma.

Lemma 5.7. Suppose that $n$ is even and $\alpha=\left(a_{1}, \ldots, a_{k}\right) \vDash n$. Then

$$
H_{+}(\alpha)= \begin{cases}2^{n-k_{o}(\alpha)} C\left(1, k_{o}(\alpha) / 2-1\right) & \text { if } a_{1} \text { and } a_{k} \text { are odd } \\ 2^{n} & \text { if } \alpha=(n), \\ 0 & \text { if } a_{1} \text { or } a_{k} \text { is even and } \alpha \neq(n) .\end{cases}
$$

Proof. We will consider the cases $\alpha=(n)$ and $\alpha \neq(n)$ separately. In this proof, the notation $\gamma i, \tilde{\gamma}$, and $\lfloor\beta / 2\rfloor$ will have the same meaning as in the proof of Lemma [5.6.

Case 1. Suppose that $\alpha=(n)$. Using the fact that $p_{+}(\gamma i)=1+u(\gamma)$, a calculation similar to the first part of the proof of Case 1 in Lemma 5.6 yields

$$
H_{+}((n))=C(0, n / 2)-\sum_{i=1}^{n-1} \sum_{b=0}^{\lfloor(n-i) / 2\rfloor}(-1)^{b} C(b+1, n / 2-b-1) \sum_{\substack{\gamma \neq n-i \\ \gamma(\gamma)=b}}(-1)^{k(\gamma)} \text {. }
$$


Make the substitution $j=\lfloor(n-i) / 2\rfloor$ and apply Lemma 5.4 (b) to get

$$
\begin{aligned}
H_{+}((n)) & =C(0, n / 2)+\sum_{j=0}^{n / 2-1} \sum_{b=0}^{j}\left(\begin{array}{l}
j \\
b
\end{array}\right) C(b+1, n / 2-b-1) \\
& =C(0, n / 2)+\sum_{b=0}^{n / 2-1} C(b+1, n / 2-b-1) \sum_{j=b}^{n / 2-1}\left(\begin{array}{l}
j \\
b
\end{array}\right) \\
& =C(0, n / 2)+\sum_{b=0}^{n / 2-1} C(b+1, n / 2-b-1)\left(\begin{array}{c}
n / 2 \\
b+1
\end{array}\right) .
\end{aligned}
$$

The last step uses the basic identity $\sum_{j=b}^{n / 2-1}\left(\begin{array}{l}j \\ b\end{array}\right)=\left(\begin{array}{l}n / 2 \\ b+1\end{array}\right)$. We have shown that $H_{+}((n))=$ $\sum_{b=0}^{n / 2}\left(\begin{array}{c}n / 2 \\ b\end{array}\right) C(b, n / 2-b)$, which equals $2^{n}$ by Lemma 5.5.

Case 2. Suppose that $\alpha \neq(n)$. Using the fact that $p_{+}(\beta \gamma)=1+u(\beta)+u(\tilde{\gamma})$ for non-empty compositions $\beta$ and $\gamma$, by calculations similar to (22) and (23), we have

$$
H_{+}(\alpha)=(-1)^{a_{k}} 2^{2\left\lfloor a_{k} / 2\right\rfloor} \sum_{\beta \geq \alpha_{k-1}}(-1)^{k(\beta)+u(\beta)+1} C\left(u(\beta)+1, n / 2-\left\lfloor a_{k} / 2\right\rfloor-u(\beta)-1\right)
$$

if $a_{k}$ is odd, and $H_{+}(\alpha)=0$ if $a_{k}$ is even.

Assume from now on that $a_{k}$ is odd. Note that $u(\delta \gamma)=u(\delta)+v(\gamma)$ for non-empty compositions, $\delta$ and $\gamma$. Using (26) as the base case, an inductive calculation similar to the proof of (24) shows that for $0 \leq i \leq k-1$,

$$
H_{+}(\alpha)=(-1)^{\left|\alpha^{i}\right|} 2^{2\left\lfloor\alpha^{i} / 2\right\rfloor} \sum_{\beta \geq \alpha_{i}}(-1)^{k(\beta)+u(\beta)+1} C\left(u(\beta)+1, n / 2-\left\lfloor\alpha^{i} / 2\right\rfloor-u(\beta)-1\right) .
$$

The formula in the lemma follows by setting $i=0$ in (27).

Proof of Theorem 5.1. Let $\rho, \psi: \mathcal{Q} S y m \rightarrow \mathbb{k}$ be the linear maps defined by the proposed formula for $\zeta_{+}$and $\zeta_{-}$, respectively. In view of (17), to conclude $\rho=\zeta_{+}$and $\psi=\zeta_{-}$, it suffices to show that

$$
\begin{gathered}
\sum_{\beta \geq \alpha}(-1)^{k(\beta)-k(\alpha)} \psi\left(F_{\beta}\right)=\zeta_{-}\left(M_{\alpha}\right) \quad \text { for } \alpha \vDash n ; \\
\sum_{\beta \geq \alpha}(-1)^{k(\beta)-k(\alpha)} \rho\left(F_{\beta}\right)=\zeta_{+}\left(M_{\alpha}\right) \quad \text { for } \alpha \vDash n, n \text { even. }
\end{gathered}
$$

According to (7), we can rewrite (28) as

$$
\begin{aligned}
& \frac{(-1)^{k(\alpha)}}{2^{2\lfloor n / 2\rfloor}} \sum_{\beta \geq \alpha}(-1)^{k(\beta)+p_{-}(\beta)} C\left(p_{-}(\beta),\lfloor n / 2\rfloor-p_{-}(\beta)\right) \\
& = \begin{cases}\frac{(-1)^{k_{e}(\alpha)}}{2^{2\left\lfloor k_{o}(\alpha) / 2\right\rfloor}} C\left(0,\left\lfloor k_{o}(\alpha) / 2\right\rfloor\right) & \text { if } a_{k} \text { is odd } \\
0 & \text { if } a_{k} \text { is even. }\end{cases}
\end{aligned}
$$


Note that the left-hand side of (30) equals $(-1)^{k(\alpha)+1} H_{-}(\alpha) / 2^{2\lfloor n / 2\rfloor}$. Furthermore, using the facts $k(\alpha)+k_{e}(\alpha) \equiv k_{o}(\alpha) \equiv n(\bmod 2)$ and $\lfloor n / 2\rfloor-\left\lfloor k_{o}(\alpha) / 2\right\rfloor=\left(n-k_{o}(\alpha)\right) / 2$, the first alternative in (30) reduces to the equation $H_{-}(\alpha)=(-1)^{n-1} 2^{n-k_{o}(\alpha)} C\left(0,\left\lfloor k_{o}(\alpha) / 2\right\rfloor\right)$; the second alternative is equivalent to $H_{-}(\alpha)=0$. These formulas for $H_{-}(\alpha)$ agree with the ones from Lemma 5.6. This proves (30) and hence (28).

By similar considerations, (29) reduces to the formulas for $H_{+}(\alpha)$ given in Lemma 5.7 .

Remark 5.8. The fact that formulas (18) and (19) define characters is not obvious, and consequences are derived in Section 7 Assuming this, however, the fact that $\zeta_{-}$is odd (which we know from Theorem 3.2) can be seen in the light of (18) as follows. First, since $\zeta_{-}$is a character, $\left(\zeta_{-}\right)^{-1}=\zeta_{-} \circ S$. For any composition $\alpha \vDash n$, the antipode of $\mathcal{Q} S y m$ is given by [17, Corollaire 4.20]

$$
S\left(F_{\alpha}\right)=(-1)^{n} F_{\alpha^{\prime}},
$$

where the ribbon diagram of the conjugate composition $\alpha^{\prime}$ is obtained by reflecting the ribbon diagram of $\alpha$ across the line $y=x$. For instance, if $\alpha=(2,3,1,2,2)$ then $\alpha^{\prime}=$ $(1,2,3,1,2,1)$, as illustrated below:
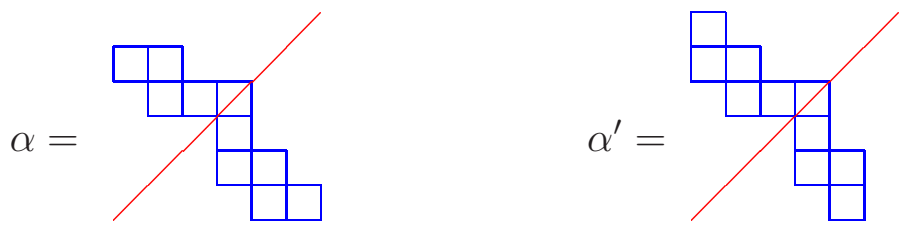

It is clear that the number of upper corners in the ribbon diagrams of $\alpha$ and $\alpha^{\prime}$ are the same, so $p_{-}(\alpha)=p_{-}\left(\alpha^{\prime}\right)$. Therefore, by (18),

$$
\left(\zeta_{-}\right)^{-1}\left(F_{\alpha}\right)=\left(\zeta_{-} \circ S\right)\left(F_{\alpha}\right)=(-1)^{n} \zeta_{-}\left(F_{\alpha^{\prime}}\right)=(-1)^{n} \zeta_{-}\left(F_{\alpha}\right)=\bar{\zeta}_{-}\left(F_{\alpha}\right),
$$

which shows that $\zeta_{-}$is odd. On the other hand, expressing this condition in the form $\bar{\zeta}_{-} \zeta_{-}=\epsilon$ leads to interesting identities involving bivariate Catalan numbers; see Section 6 .

Remark 5.9. Formula (19) reveals an analogous property of the character $\zeta_{+}$, which is not obvious from its definition. Recall that if $\alpha=\left(a_{1}, \ldots, a_{k}\right)$ then $\tilde{\alpha}=\left(a_{k}, \ldots, a_{1}\right)$ denotes its reversal. The ribbon diagram of $\tilde{\alpha}$ is obtained by reflecting the ribbon diagram of $\alpha$ across the line $y=-x$. Consider the linear map $T: \mathcal{Q}$ Sym $\rightarrow \mathcal{Q}$ Sym defined by

$$
T\left(F_{\alpha}\right)=F_{\tilde{\alpha}} .
$$

Since $\alpha \mapsto \tilde{\alpha}$ preserves refinements, the map $T$ is also given by

$$
T\left(M_{\alpha}\right)=M_{\tilde{\alpha}}
$$

From either formula it follows easily that $T$ is an antimorphism of coalgebras, a morphism of algebras, and an involution (properties which are shared by the antipode $S$ of $\mathcal{Q} S y m$ ). Moreover, we have

$$
\zeta_{+} \circ T=\zeta_{+}
$$


This property follows from (19) and the fact that $p_{+}(\alpha)=p_{+}(\tilde{\alpha})$, which is obvious from (16). Another proof of this fact is given in Proposition 8.2

Remark 5.10. Let $\alpha=\left(a_{1}, \ldots, a_{k}\right)$. As mentioned in the preceding remarks,

$$
p_{-}\left(\alpha^{\prime}\right)=p_{-}(\alpha) \text { and } p_{+}(\tilde{\alpha})=p_{+}(\alpha) \text {. }
$$

We also have the following formulas:

$$
\begin{aligned}
& p_{-}(\tilde{\alpha})= \begin{cases}p_{-}(\alpha) & \text { if } a_{1}=a_{k}=1 \text { or } a_{1}, a_{k} \neq 1, \\
p_{-}(\alpha)-1 & \text { if } a_{1} \neq 1 \text { and } a_{k}=1, \\
p_{-}(\alpha)+1 & \text { if } a_{1}=1 \text { and } a_{k} \neq 1 ;\end{cases} \\
& p_{+}\left(\alpha^{\prime}\right)= \begin{cases}p_{+}(\alpha) & \text { if exactly one of } a_{1} \text { and } a_{k} \text { is } 1, \\
p_{+}(\alpha)-1 & \text { if both } a_{1} \text { and } a_{k} \text { are } 1, \\
p_{+}(\alpha)+1 & \text { if neither } a_{1} \text { nor } a_{k} \text { is } 1 .\end{cases}
\end{aligned}
$$

These formulas follow easily from (15) and (16), but they can also be visualized in terms of ribbon diagrams. We illustrate the second alternative of (35) below, for $\alpha=(1,3,1,2,1)$. Recall that $p_{+}(\alpha)$ is the number of upper corners in the augmented ribbon diagram of $\alpha$.

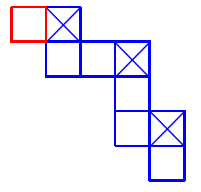

$$
p_{+}(\alpha)=3
$$

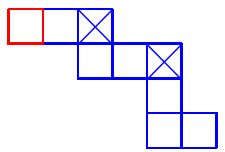

$$
p_{+}\left(\alpha^{\prime}\right)=2
$$

The statistics $p_{-}(\tilde{\alpha})$ and $p_{+}\left(\alpha^{\prime}\right)$ enter in some of the formulas in Section 8 ,

\section{Application: Identities for bivariate Catalan num- bers}

The first applications we propose stem from evaluating products of characters on the basis $F_{\alpha}$ of $\mathcal{Q}$ Sym. This requires knowledge of the coproduct of $\mathcal{Q}$ Sym on this basis. Consider all ways of cutting the ribbon diagram of $\alpha$ into two pieces along the common boundary of two squares. We include the two trivial cuts along the first and last edges. Thus, if $\alpha$ is a composition of $n$, there are $n+1$ ways of cutting its diagram. For $\alpha=(2,3,1,2,2)$, two non-trivial cuts are shown below.

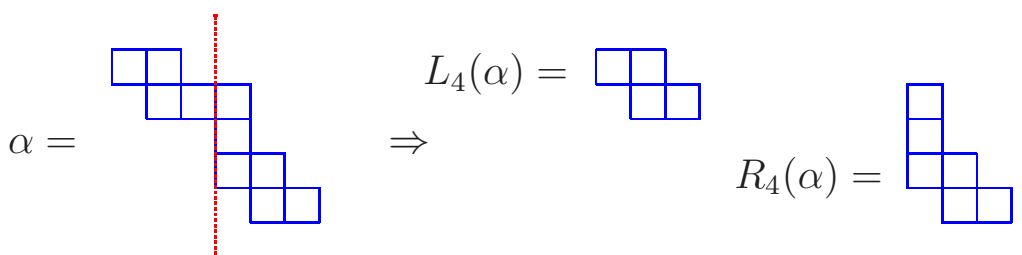




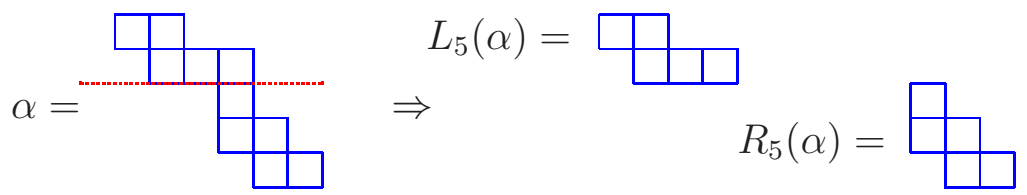

Label the edges between adjacent squares from 0 to $n$ from left to right and top to bottom, including the first and last edges. Let $L_{i}(\alpha)$ and $R_{i}(\alpha)$ be the compositions whose ribbon diagrams are the resulting pieces after cutting the ribbon diagram of $\alpha$ along edge $i$, with $L_{i}(\alpha)$ corresponding to the left piece and $R_{i}(\alpha)$ to the right one. The two trivial cuts result in $L_{0}(\alpha)=(), R_{0}(\alpha)=\alpha$, and $L_{n}(\alpha)=\alpha, R_{n}(\alpha)=()$. The coproduct of $\mathcal{Q} S y m$ is [17, Corollaire 4.17]

$$
\Delta\left(F_{\alpha}\right)=\sum_{i=0}^{n} F_{L_{i}(\alpha)} \otimes F_{R_{i}(\alpha)}
$$

The counit is

$$
\epsilon\left(F_{\alpha}\right)= \begin{cases}1 & \text { if } \alpha=() \\ 0 & \text { otherwise }\end{cases}
$$

We abbreviate

$$
\begin{array}{ll}
\ell_{+}^{i}(\alpha):=p_{+}\left(L_{i}(\alpha)\right), & r_{+}^{i}(\alpha):=p_{+}\left(R_{i}(\alpha)\right), \\
\ell_{-}^{i}(\alpha):=p_{-}\left(L_{i}(\alpha)\right), & r_{-}^{i}(\alpha):=p_{-}\left(R_{i}(\alpha)\right) .
\end{array}
$$

Proposition 6.1. For any composition $\alpha$ of a positive integer $n$,

$$
\begin{aligned}
& \sum_{j=0}^{\lfloor n / 2\rfloor}(-1)^{\ell_{+}^{2 j}(\alpha)+r_{-}^{2 j}(\alpha)} C\left(\ell_{+}^{2 j}(\alpha), j-\ell_{+}^{2 j}(\alpha)\right) C\left(r_{-}^{2 j}(\alpha),\lfloor n / 2\rfloor-j-r_{-}^{2 j}(\alpha)\right) \\
& = \begin{cases}4^{\lfloor n / 2\rfloor} & \text { if } \alpha=(n), \\
0 & \text { otherwise. }\end{cases} \\
& \sum_{i=0}^{n} \frac{(-1)^{\ell_{-}^{i}(\alpha)+r_{-}^{i}(\alpha)+i}}{4^{\left\lfloor\frac{i}{2}\right\rfloor+\left\lfloor\frac{n-i}{2}\right\rfloor} C\left(\ell_{-}^{i}(\alpha),\lfloor i / 2\rfloor-\ell_{-}^{i}(\alpha)\right) C\left(r_{-}^{i}(\alpha),\lfloor(n-i) / 2\rfloor-r_{-}^{i}(\alpha)\right)=0 .}
\end{aligned}
$$

Proof. Since $\zeta_{+}$and $\zeta_{-}$are the even and odd parts of $\zeta$, we have

$$
\zeta\left(F_{\alpha}\right)=\left(\zeta_{+} \zeta_{-}\right)\left(F_{\alpha}\right)=\sum_{i=0}^{n} \zeta_{+}\left(F_{L_{i}(\alpha)}\right) \zeta_{-}\left(F_{R_{i}(\alpha)}\right) .
$$

Note that $\left|L_{i}(\alpha)\right|=i$. Hence, by (19), only the terms corresponding to even $i$ contribute to this sum. We evaluate these terms using (18), (19), and (2). The power of 2 that results in the denominator is constant and equal to $2\lfloor n / 2\rfloor$. Identity (37) follows.

Identity (38) follows similarly from $\left(\bar{\zeta}_{-} \zeta_{-}\right)\left(F_{\alpha}\right)=\epsilon\left(F_{\alpha}\right)=0$. 
Various identities for bivariate Catalan numbers may be obtained through special choices of $\alpha$ in Proposition 6.1] We discuss two particularly nice cases.

The first case involves central Catalan numbers, by which we mean the numbers

$$
\begin{array}{rlrl}
C_{1}(h):=\frac{1}{2} C(2 h+1, h+1), & C_{2}(h) & :=\frac{1}{2} C(2 h, h+1), \\
C_{3}(h):=\frac{1}{2} C(2 h, h), & C_{4}(h):=\frac{1}{2} C(2 h+1, h) .
\end{array}
$$

It follows from (11) that

$$
\begin{array}{rrr}
C_{1}(h)=\left(\begin{array}{c}
4 h+2 \\
h
\end{array}\right), & C_{2}(h)=\frac{2 h+1}{4 h+1}\left(\begin{array}{c}
4 h+1 \\
h
\end{array}\right), \\
C_{3}(h)=\frac{1}{2}\left(\begin{array}{c}
4 h \\
h
\end{array}\right), & C_{4}(h)=\left(\begin{array}{c}
4 h+1 \\
h
\end{array}\right) .
\end{array}
$$

We have the following wonderful convolution formulas for central Catalan numbers. Note that on each side of each equation, the total sum of the subindices $r$ in $C_{r}$ is constant (equal to 6,7 , and 8 in each case). Ira Gessel has shown us how one may derive these identities via generating functions [13].

Corollary 6.2. For any positive integer $h$,

$$
\begin{aligned}
& \sum_{j=0}^{h} C_{3}(j) C_{3}(h-j)=2 \sum_{j=0}^{h-1} C_{2}(j) C_{1}(h-1-j), \\
& \sum_{j=0}^{h} C_{3}(j) C_{4}(h-j)=\sum_{j=0}^{h} C_{2}(j) C_{3}(h-j)+\sum_{j=0}^{h-1} C_{1}(j) C_{1}(h-1-j), \\
& \sum_{j=0}^{h} C_{4}(j) C_{4}(h-j)=2 \sum_{j=0}^{h} C_{3}(j) C_{1}(h-j) .
\end{aligned}
$$

Proof. Let $k$ be a positive integer and $\alpha:=(2,1)^{k}=(2,1,2,1, \ldots, 2,1)$. Formula (36) gives

$$
\Delta\left(F_{\alpha}\right)=\sum_{i=0}^{k} F_{(2,1)^{i}} \otimes F_{(2,1)^{k-i}}+\sum_{i=0}^{k-1} F_{(2,1)^{i}, 2} \otimes F_{1,(2,1)^{k-1-i}}+\sum_{i=0}^{k-1} F_{(2,1)^{i}, 1} \otimes F_{1,1,(2,1)^{k-1-i}} .
$$

Equation (37) then leads to

$$
\sum_{\substack{i=0 \\ i \text { even }}}^{k} C(i, i / 2) C(k-i,\lfloor(k-i) / 2\rfloor)=\sum_{i=0}^{k-1} C(i,\lfloor i / 2\rfloor+1) C(k-1-i,\lfloor k / 2\rfloor-\lfloor i / 2\rfloor) .
$$

When $k=2 h$ this is (40); when $k=2 h+1$ this is (41).

Similarly, equation (38) for $\alpha=(2,1)^{k}$ leads to (42) when $k=2 h$ (and to a trivial identity when $k=2 h+1$ ). 
The second application was suggested by one of the referees. In (44), we adopt the convention that $C(1,-1)=-1$. This is in agreement with (69).

Corollary 6.3. For any positive integers $m$ and $n$,

$$
\begin{aligned}
\sum_{j=0}^{m-1} C(0, j) C(n, m-1-j) & =\sum_{j=0}^{n-1} C(0, j) C(m, n-1-j), \\
\sum_{j=0}^{m-1} C(0, j) C(n, m-j) & =\sum_{j=0}^{n} C(1, j-1) C(m, n-j) .
\end{aligned}
$$

Proof. Equations (43) and (44) follow from (37) applied to $\alpha=\left(2 m, 2^{n}, 1\right)$ and $\alpha=$ $\left(2 m, 2^{n}\right)$, respectively.

More identities may be derived from (18) and (19) by imposing the fact that $\zeta_{-}$and $\zeta_{+}$are morphisms of algebras. The multiplication of two basis elements $F_{\alpha}$ and $F_{\beta}$ is most easily described in terms of permutations. It is thus convenient to work on a larger Hopf algebra $\mathcal{S} S y m$, of which $\mathcal{Q}$ Sym is a quotient. This is the object of the next section.

\section{Identities for bivariate Catalan numbers via the Hopf algebra of permutations}

The Hopf algebra of permutations $\mathcal{S} S y m$ has a linear basis $\left\{F_{\sigma}\right\}$ indexed by permutations $\sigma \in S_{n}, n \geq 0$. The multiplication of two basis elements is as follows. Given $\sigma \in$ $S_{n}$ and $\tau \in S_{m}$, let $\mathrm{S}(\sigma, \tau)$ be the set of all shuffles of the words $\sigma(1), \ldots, \sigma(n)$ and $n+\tau(1), \ldots, n+\tau(m)$. Then

$$
F_{\sigma} \cdot F_{\tau}=\sum_{\rho \in \mathrm{S}(\sigma, \tau)} F_{\rho}
$$

For example,

$$
\begin{aligned}
F_{12} \cdot F_{312}= & F_{12534}+F_{15234}+F_{15324}+F_{15342}+F_{51234} \\
+ & F_{51324}+F_{51342}+F_{53124}+F_{53142}+F_{53412} .
\end{aligned}
$$

For more information on the Hopf algebra structure of $\mathcal{S} S y m$ see [3].

The descent set of a permutation $\sigma \in S_{n}$ is

$$
\operatorname{Des}(\sigma):=\{i \in[n-1] \mid \sigma(i)>\sigma(i+1)\} .
$$

Let $D(\sigma)=\left(a_{1}, \ldots, a_{k}\right)$ be the composition of $n$ such that $\left\{a_{1}, a_{1}+a_{2}, \ldots, a_{1}+\cdots+a_{k-1}\right\}=$ $\operatorname{Des}(\sigma)$. The map

$$
\begin{aligned}
\mathcal{D}: \text { S Sym } & \longrightarrow \mathcal{Q S y m} \\
F_{\sigma} & \longmapsto F_{D(\sigma)}
\end{aligned}
$$


is a surjective morphism of graded Hopf algebras [17 Théorèmes 5.12, 5.13, and 5.18].

Let $\zeta_{\mathcal{S}}$ denote the pull-back of the universal character $\zeta$ of $\mathcal{Q}$ Sym via the morphism $\mathcal{D}:$

$$
\zeta_{\mathcal{S}}:=\zeta \circ \mathcal{D}
$$

According to Lemma 2.2, the even and odd parts of $\zeta_{\mathcal{S}}$ are

$$
\left(\zeta_{\mathcal{S}}\right)_{+}=\zeta_{+} \circ \mathcal{D} \text { and }\left(\zeta_{\mathcal{S}}\right)_{-}=\zeta_{-} \circ \mathcal{D} \text {. }
$$

We describe these characters directly in terms of permutations.

As in [1, consider the following two slightly differing notions of a peak set of a permutation $\sigma \in S_{n}$ :

$$
\begin{aligned}
\operatorname{Peak}^{0}(\sigma) & :=\{i \in[n-1] \mid i \neq 1, \sigma(i-1)<\sigma(i)>\sigma(i+1)\}, \\
\operatorname{Peak}(\sigma) & :=\{i \in[n-1] \mid \sigma(i-1)<\sigma(i)>\sigma(i+1)\},
\end{aligned}
$$

where we agree that $\sigma(0)=0$. For instance, if $\sigma=312546$ then $\operatorname{Peak}^{0}(\sigma)=\{4\}$ and $\operatorname{Peak}(\sigma)=\{1,4\}$. The study of peak enumeration has a long history, but the connections between peaks and quasi-symmetric functions originate in work of Stembridge 23].

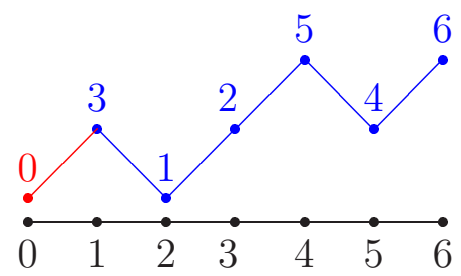

Note that $\operatorname{Peak}^{0}(\sigma)$ and $\operatorname{Peak}(\sigma)$ depend only on $\operatorname{Des}(\sigma)$. In fact,

$$
\begin{aligned}
i \in \operatorname{Peak}(\sigma) & \Longleftrightarrow i \in \operatorname{Des}(\sigma) \text { and } i-1 \notin \operatorname{Des}(\sigma), \\
i \in \operatorname{Peak}^{0}(\sigma) & \Longleftrightarrow i \in \operatorname{Peak}(\sigma) \text { and } i \neq 1 .
\end{aligned}
$$

We write $p_{-}(\sigma):=\# \operatorname{Peak}^{0}(\sigma)$ and $p_{+}(\sigma):=\# \operatorname{Peak}(\sigma)$. Let $\alpha:=D(\sigma)$. It follows from (15), (16), and the above that

$$
p_{-}(\sigma)=p_{-}(\alpha) \quad \text { and } \quad p_{+}(\sigma)=p_{+}(\alpha) .
$$

Proposition 7.1. Let $\sigma \in S_{n}$ be a permutation. Then

$$
\begin{aligned}
& \left(\zeta_{\mathcal{S}}\right)_{-}\left(F_{\sigma}\right)=\frac{(-1)^{p_{-}(\sigma)}}{2^{2\lfloor n / 2\rfloor} C\left(p_{-}(\sigma),\lfloor n / 2\rfloor-p_{-}(\sigma)\right) .} \\
& \left(\zeta_{\mathcal{S}}\right)_{+}\left(F_{\sigma}\right)= \begin{cases}\frac{(-1)^{p_{+}(\sigma)}}{2^{n}} C\left(p_{+}(\sigma), n / 2-p_{+}(\sigma)\right) & \text { if } n \text { is even, } \\
0 & \text { if } n \text { is odd. }\end{cases}
\end{aligned}
$$

Proof. This follows at once from Theorem [5.] together with (46)), (47) and (48)). 
We may now deduce the identities for bivariate Catalan numbers announced at the end of Section 6.

Corollary 7.2. For any non-negative integer n,

$$
\sum_{\sigma \in S_{n}}(-1)^{p_{-}(\sigma)} C\left(p_{-}(\sigma),\lfloor n / 2\rfloor-p_{-}(\sigma)\right)=2^{2\lfloor n / 2\rfloor},
$$

and for any even positive integer $n$,

$$
\sum_{\sigma \in S_{n}}(-1)^{p_{+}(\sigma)} C\left(p_{+}(\sigma), n / 2-p_{+}(\sigma)\right)=0
$$

Proof. Consider the $n$-th power of the basis element indexed by the permutation $1 \in S_{1}$ in the algebra $\mathcal{S} S y m$. Since $S(1,1, \ldots, 1)=S_{n}$, [45) gives

$$
\left(F_{1}\right)^{n}=\sum_{\sigma \in S_{n}} F_{\sigma}
$$

Applying $\left(\zeta_{\mathcal{S}}\right)_{-}$to both sides, using that it is a morphism of algebras and (49), we deduce (51). Similarly, applying $\left(\zeta_{\mathcal{S}}\right)_{+}$we obtain (52).

Let $1_{n}$ denote the identity permutation in $S_{n}$. Note that $\mathrm{S}\left(1_{n}, 1_{m}\right)$ is the set of those permutations $\sigma \in S_{n+m}$ such that

$$
\sigma^{-1}(1)<\cdots<\sigma^{-1}(n), \sigma^{-1}(n+1)<\cdots<\sigma^{-1}(n+m) .
$$

Corollary 7.3. Let $n, m$ be non-negative integers. Then

$$
\begin{aligned}
& \sum_{\sigma \in \mathrm{S}\left(1_{n}, 1_{m}\right)}(-1)^{p_{-}(\sigma)} C\left(p_{-}(\sigma),\lfloor(n+m) / 2\rfloor-p_{-}(\sigma)\right) \\
& = \begin{cases}C(0,\lfloor n / 2\rfloor) C(0,\lfloor m / 2\rfloor) & \text { if } n \text { or } m \text { is even, } \\
4 C(0,\lfloor n / 2\rfloor) C(0,\lfloor m / 2\rfloor) & \text { if } n \text { and } m \text { are odd. }\end{cases} \\
& \sum_{\sigma \in \mathrm{S}\left(1_{n}, 1_{m}\right)}(-1)^{p_{+}{ }^{(\sigma)} C\left(p_{+}(\sigma),(n+m) / 2-p_{+}(\sigma)\right)} \\
& = \begin{cases}C(0, n / 2) C(0, m / 2) & \text { if } n \text { and } m \text { are even, } \\
0 & \text { if } n \text { and } m \text { are odd. }\end{cases}
\end{aligned}
$$

Proof. By (45),

$$
F_{1_{n}} \cdot F_{1_{m}}=\sum_{\sigma \in \mathrm{S}\left(1_{n}, 1_{m}\right)} F_{\sigma}
$$

Applying $\left(\zeta_{\mathcal{S}}\right)_{-}$we deduce (153) and applying $\left(\zeta_{\mathcal{S}}\right)_{+}$we deduce (54). 


\section{Inverses of canonical characters and more applica- tions}

The set of characters of a Hopf algebra $\mathcal{H}$ is a group under the convolution product (Section 1). The inverse of a character $\varphi$ is $\varphi \circ S$, where $S$ is the antipode of $\mathcal{H}$.

Proposition 8.1. The inverse of the universal character $\zeta$ of $\mathcal{Q} S y m$ is explicitly given by

$$
\zeta^{-1}\left(M_{\alpha}\right)=(-1)^{k(\alpha)}, \quad \zeta^{-1}\left(F_{\alpha}\right)= \begin{cases}(-1)^{|\alpha|} & \text { if } \alpha=(1,1, \ldots, 1), \\ 0 & \text { if not. }\end{cases}
$$

Proof. This follows at once from the explicit formulas for the antipode of $\mathcal{Q}$ Sym (12) and (31).

Inverting the canonical decomposition $\zeta=\zeta_{+} \zeta_{-}$we obtain

$$
\zeta^{-1}=\left(\zeta_{-}\right)^{-1}\left(\zeta_{+}\right)^{-1}=\left(\zeta_{+}\right)^{-1}\left(\zeta_{+}\left(\zeta_{-}\right)^{-1}\left(\zeta_{+}\right)^{-1}\right)=\left(\zeta_{+}\right)^{-1}\left(\zeta_{+} \bar{\zeta}_{-}\left(\zeta_{+}\right)^{-1}\right)
$$

The set of even characters is a subgroup of the group of characters, and the set of odd characters is closed under conjugation by even characters [2, Proposition 1.7]. In particular, $\left(\zeta_{+}\right)^{-1}$ is even and $\zeta_{+} \bar{\zeta}_{-}\left(\zeta_{+}\right)^{-1}$ is odd. According to Lemma 2.1, these are the even and odd parts of $\zeta^{-1}$ :

$$
\left(\zeta^{-1}\right)_{+}=\left(\zeta_{+}\right)^{-1} \text { and }\left(\zeta^{-1}\right)_{-}=\zeta_{+} \bar{\zeta}_{-}\left(\zeta_{+}\right)^{-1}
$$

We provide explicit descriptions for these characters below. First, we analyze the behavior of the map $T: \mathcal{Q}$ Sym $\rightarrow \mathcal{Q}$ Sym (Remark 5.9) with respect to the canonical decomposition of $\zeta$.

Proposition 8.2. We have

$$
\zeta_{+} \circ T=\zeta_{+} \text {and } \zeta_{-} \circ T=\left(\left(\zeta^{-1}\right)_{-}\right)^{-1}
$$

Proof. Both $S$ and $T$ are antimorphism of coalgebras and morphisms of algebras $\mathcal{Q} S y m \rightarrow$ $\mathcal{Q} S y m$. In addition, $\zeta \circ S=\zeta^{-1}$ and $\zeta \circ T=\zeta$; the latter being an immediate consequence of (2) and (32). Therefore, $T \circ S: \mathcal{Q} S y m \rightarrow \mathcal{Q} S y m$ is a morphism of Hopf algebras such that $\zeta \circ T \circ S=\zeta^{-1}$. According to Lemma 2.2 we have

$$
\left(\zeta^{-1}\right)_{+}=\zeta_{+} \circ T \circ S \text { and }\left(\zeta^{-1}\right)_{-}=\zeta_{-} \circ T \circ S .
$$

Composing with $S$ we find $\zeta_{+} \circ T=\left(\zeta^{-1}\right)_{+} \circ S=\left(\left(\zeta^{-1}\right)_{+}\right)^{-1}=\zeta_{+}$, by (156), and $\zeta_{-} \circ T=$ $\left(\zeta^{-1}\right)_{-} \circ S=\left(\left(\zeta^{-1}\right)_{-}\right)^{-1}$. 
Remark 8.3. The fact that $\zeta_{+} \circ T=\zeta_{+}$was observed in Remark 5.9. The fact that $\zeta_{-} \circ T=\left(\left(\zeta^{-1}\right)_{-}\right)^{-1}$ may be rewritten as follows:

$$
\left(\zeta^{-1}\right)_{-}=\bar{\zeta}_{-} \circ T
$$

Indeed, any morphism (or antimorphism) preserves inverses, so

$$
\left(\zeta^{-1}\right)_{-}=\left(\zeta_{-} \circ T\right)^{-1}=\left(\zeta_{-}\right)^{-1} \circ T=\bar{\zeta}_{-} \circ T,
$$

since $\zeta_{-}$is odd. We use this formula to calculate $\left(\zeta^{-1}\right)_{-}$below.

Theorem 8.4. Let $\alpha=\left(a_{1}, \ldots, a_{k}\right)$ be a composition of a non-negative integer $n$. Then

$$
\begin{aligned}
& \left(\zeta^{-1}\right)_{-}\left(M_{\alpha}\right)= \begin{cases}\frac{(-1)^{k(\alpha)}}{2^{2\left\lfloor k_{o}(\alpha) / 2\right\rfloor}} C\left(0,\left\lfloor k_{o}(\alpha) / 2\right\rfloor\right) & \text { if } a_{1} \text { is odd, } \\
0 & \text { if } a_{1} \text { is even. }\end{cases} \\
& \left(\zeta^{-1}\right)_{+}\left(M_{\alpha}\right)= \begin{cases}\frac{(-1)^{k(\alpha)}}{2^{k_{o}(\alpha)}} C\left(0, k_{o}(\alpha) / 2\right) & \text { if } n \text { is even, } \\
0 & \text { if } n \text { is odd; }\end{cases}
\end{aligned}
$$

Proof. According to (58) and (33),

$$
\left(\zeta^{-1}\right)_{-}\left(M_{\alpha}\right)=\bar{\zeta}_{-}\left(T\left(M_{\alpha}\right)\right)=(-1)^{n} \zeta_{-}\left(M_{\tilde{\alpha}}\right),
$$

which we can evaluate with (77). To see that this results in (59), note that since $\tilde{\alpha}$ is the reversal of $\alpha$, the last part of $\tilde{\alpha}$ is $a_{1}, k_{o}(\tilde{\alpha})=k_{o}(\alpha)$, and by (6)

$$
n+k_{e}(\tilde{\alpha})=n+k_{e}(\alpha) \equiv k_{o}(\alpha)+k_{e}(\alpha)=k(\alpha) .
$$

To settle the remaining identity we give a direct argument. Let $\varphi: \mathcal{Q} S y m \rightarrow \mathbb{k}$ be the linear functional defined by (60) . We show that $\zeta_{+} \varphi=\epsilon$, which implies $\varphi=\left(\zeta_{+}\right)^{-1}=$ $\left(\zeta^{-1}\right)_{+}$.

Since $\varphi\left(M_{()}\right)=1$, we have $\left(\zeta_{+} \varphi\right)\left(M_{()}\right)=1=\epsilon\left(M_{()}\right)$.

Assume from now on that $n>0$. We need to show $\left(\zeta_{+} \varphi\right)\left(M_{\alpha}\right)=0$. Write $\alpha=$ $\left(a_{1}, \ldots, a_{k}\right)$ and recall the notations $\alpha_{i}$ and $\alpha^{i}$ from (3).

If $n$ is odd then for each $i$ one of $\left|\alpha_{i}\right|$ and $\left|\alpha^{i}\right|$ is odd, so every term in the expansion (3) of $\left(\zeta_{+} \varphi\right)\left(M_{\alpha}\right)$ is 0 , by (8) and (60). Assume from now on that $n$ is even.

If $k=1$ (i.e., $\alpha=(n)$ ), then $\left(\zeta_{+} \varphi\right)\left(M_{(n)}\right)=\varphi\left(M_{(n)}\right)+\zeta_{+}\left(M_{(n)}\right)=-1+1=0$.

If $k>1$ and $a_{1}$ is even, then $\varphi\left(M_{\alpha}\right)=-\varphi\left(M_{\alpha^{1}}\right)$ (since $\alpha^{1}$ has one less part than $\alpha$ and the same number of odd parts), and $\zeta_{+}\left(M_{\alpha_{i}}\right)=0$ when $i>1$ by (8). Thus,

$$
\left(\zeta_{+} \varphi\right)\left(M_{\alpha}\right)=\varphi\left(M_{\alpha}\right)+\zeta_{+}\left(M_{\left(a_{1}\right)}\right) \varphi\left(M_{\alpha^{1}}\right)=\varphi\left(M_{\alpha}\right)+\varphi\left(M_{\alpha^{1}}\right)=0 .
$$


Suppose finally that $k>1$ and $a_{1}$ is odd. Using (3) and (8) we compute

$$
\begin{aligned}
\left(\zeta_{+} \varphi\right)\left(M_{\alpha}\right) & =\frac{(-1)^{k(\alpha)}}{2^{k_{o}(\alpha)}} B\left(\frac{k_{o}(\alpha)}{2}\right)+\sum_{\substack{1 \leq i \leq k \\
a_{i} \text { odd } \\
\left|\alpha_{i}\right| \text { even }}} \frac{(-1)^{k_{e}\left(\alpha_{i}\right)+1}}{2^{k_{o}\left(\alpha_{i}\right)-1}} C\left(\frac{k_{o}\left(\alpha_{i}\right)}{2}-1\right) \frac{(-1)^{k\left(\alpha^{i}\right)}}{2^{k_{o}\left(\alpha^{i}\right)}} B\left(\frac{k_{o}\left(\alpha^{i}\right)}{2}\right) \\
& =\frac{(-1)^{k}}{2^{k_{o}(\alpha)}} B\left(\frac{k_{o}(\alpha)}{2}\right)+\frac{(-1)^{k+1}}{2^{k_{o}(\alpha)-1}} \sum_{\substack{1 \leq i \leq k \\
a_{i} \text { odd } \\
\left|\alpha_{i}\right| \text { even }}} C\left(\frac{k_{o}\left(\alpha_{i}\right)}{2}-1\right) B\left(\frac{k_{o}\left(\alpha^{i}\right)}{2}\right) .
\end{aligned}
$$

To combine the signs in the last step we used $k=k(\alpha) \equiv k_{e}\left(\alpha_{i}\right)+k\left(\alpha^{i}\right) \bmod 2$, which holds by (6) and since $\left|\alpha_{i}\right|,\left|\alpha^{i}\right|$, and $|\alpha|$ are all even. The argument may now be completed as in the proof of Theorem 3.2, Case 5

We present some applications. The first one is an identity which appears in [19, Section 4.2, Example 2] (near the bottom of page 130).

Corollary 8.5. For any positive integer $m$,

$$
\sum_{j=0}^{m-1} 2^{2 m-2 j-1} C(j)=2^{2 m}-\left(\begin{array}{c}
2 m \\
m
\end{array}\right) .
$$

Proof. We have $\zeta^{-1}=\left(\zeta^{-1}\right)_{+}\left(\zeta^{-1}\right)_{-}=\left(\zeta_{+}\right)^{-1}\left(\zeta^{-1}\right)_{-}$, so $\left(\zeta^{-1}\right)_{-}=\zeta_{+} \zeta^{-1}$. We evaluate both sides on $M_{\alpha}$ with $\alpha=(1,1, \ldots, 1) \vDash 2 m$. Equations (8), (55), and (59) lead to

$$
\frac{1}{2^{2 m}} C(0, m)=1+\sum_{\substack{i=2 \\ i \text { even }}}^{2 m} \frac{-1}{2^{i}} C(1, i / 2-1) .
$$

Letting $j=2 i+2$ we obtain ([6]).

The following identity is analogous to (13).

Corollary 8.6. For any composition $\beta$ of an even integer,

$$
\sum_{\substack{\alpha \leq \beta \\
a_{1}, a_{k} \text { odd }}}(-1)^{k_{e}(\alpha)} 2^{k_{o}(\beta)-k_{o}(\alpha)+1} C\left(k_{o}(\alpha) / 2-1\right)=2^{k_{o}(\beta)}-\left(\begin{array}{c}
k_{o}(\beta) \\
k_{o}(\beta) / 2
\end{array}\right)
$$

the sum being over those compositions $\alpha=\left(a_{1}, \ldots, a_{k}\right)$ whose first and last part are odd and which are refined by $\beta$.

Proof. By (56),

$$
\zeta_{+} \circ S=\left(\zeta_{+}\right)^{-1}=\left(\zeta^{-1}\right)_{+} .
$$

Therefore, for any composition $\beta$ of $n$ we have, by (12),

$$
(-1)^{k(\beta)} \sum_{\alpha \leq \tilde{\beta}} \zeta_{+}\left(M_{\alpha}\right)=\left(\zeta^{-1}\right)_{+}\left(M_{\beta}\right)
$$


Hence, by (8) and (60), and since $n$ is even,

$$
(-1)^{k(\beta)}+(-1)^{k(\beta)} \sum_{\substack{\alpha \leq \tilde{\beta} \\ a_{1}, a_{h} \text { odd }}} \frac{(-1)^{k_{e}(\alpha)+1}}{2^{k_{o}(\alpha)}} C\left(1, k_{o}(\alpha) / 2-1\right)=\frac{(-1)^{k(\beta)}}{2^{k_{o}(\beta)}} C\left(0, k_{o}(\beta) / 2\right) .
$$

The above sum remains unchanged if we replace $\tilde{\beta}$ by $\beta$. Multiplying by $2^{k_{o}(\beta)}$ gives (62).

On the fundamental basis, the even and odd parts of $\zeta^{-1}$ are most easily described in terms of the statistics $p_{+}\left(\alpha^{\prime}\right)$ and $p_{-}(\tilde{\alpha})$ (Remark 5.10). They may be described directly in terms of $p_{+}(\alpha)$ and $p_{-}(\alpha)$ by means of formulas (34) and (35).

Theorem 8.7. Let $\alpha=\left(a_{1}, \ldots, a_{k}\right)$ be a composition of a non-negative integer $n$. Then

$$
\begin{aligned}
\left(\zeta^{-1}\right)_{-}\left(F_{\alpha}\right) & =\frac{(-1)^{n+p_{-}(\tilde{\alpha})}}{2^{2\lfloor n / 2\rfloor} C\left(p_{-}(\tilde{\alpha}),\lfloor n / 2\rfloor-p_{-}(\tilde{\alpha})\right)} \\
\left(\zeta^{-1}\right)_{+}\left(F_{\alpha}\right) & = \begin{cases}\frac{(-1)^{p_{+}\left(\alpha^{\prime}\right)}}{2^{n}} C\left(p_{+}\left(\alpha^{\prime}\right), n / 2-p_{+}\left(\alpha^{\prime}\right)\right) & \text { if } n \text { is even }, \\
0 & \text { if } n \text { is odd } .\end{cases}
\end{aligned}
$$

Proof. Assume that $n$ is even, $n \neq 0$. We have, by (31) and (56),

$$
\left(\zeta^{-1}\right)_{+}\left(F_{\alpha}\right)=\left(\zeta_{+}\right)^{-1}\left(F_{\alpha}\right)=\left(\zeta_{+} \circ S\right)\left(F_{\alpha}\right)=\zeta_{+}\left(F_{\alpha^{\prime}}\right)
$$

Formula (64) now follows from (19). Formula (63) follows similarly from (18) and (58).

As an application we derive a recursive formula for the bivariate Catalan numbers.

Corollary 8.8. For any non-negative integers $a, b$, and $c$,

$$
C(b, a+c)=4^{c} C(b, a)-\sum_{j=1}^{c} 4^{c-j} C(b+1, a+j-1) .
$$

Proof. We evaluate both sides of $\left(\zeta^{-1}\right)_{-}=\zeta_{+} \zeta^{-1}$ on $F_{\alpha}$ with

$$
\alpha=(\underbrace{1, \ldots, 1}_{2 a+1}, \underbrace{2, \ldots, 2}_{b}, \underbrace{1, \ldots, 1}_{2 c}) .
$$

According to (36),

$$
\left(\zeta_{+} \zeta^{-1}\right)\left(F_{\alpha}\right)=\sum_{i=0}^{n} \zeta_{+}\left(F_{L_{i}(\alpha)}\right) \zeta^{-1}\left(F_{R_{i}(\alpha)}\right)
$$


Several terms in this expansion vanish, in view of (19) and (55): all those for which the size of $L_{i}(\alpha)$ is odd and all those for which $R_{i}(\alpha) \neq(1, \ldots, 1)$. We are left with

$$
\begin{aligned}
& \left.\left(\zeta_{+} \zeta^{-1}\right)\left(F_{\alpha}\right)=\zeta_{+}(F_{(\underbrace{1, \ldots, 1}_{2 a+1}} \underbrace{2, \ldots, 2}_{b-1}, 1)\right) \zeta^{-1}\left(F_{(\underbrace{1, \ldots, 1}_{2 c+1}}\right) \\
& +\sum_{\substack{i=1 \\
i \text { odd }}}^{2 c-1} \zeta_{+}(F_{(\underbrace{1, \ldots, 1}_{2 a+1}}^{2, \ldots, 2} \underbrace{1, \ldots, 1}_{b}) \zeta^{-1}(\underbrace{\left(F^{1, \ldots, 1}\right.}_{i}) \\
& =\frac{(-1)^{b}}{2^{2(a+b)}} C(b, a)(-1)^{2 c+1}+\sum_{\substack{i=1 \\
i \text { odd }}}^{2 c-1} \frac{(-1)^{b+1}}{2^{2(a+b)+i+1}} C(b+1, a+(i+1) / 2-1)(-1)^{2 c-i} \\
& =\frac{(-1)^{b+1}}{2^{2(a+b)}} C(b, a)+\sum_{j=1}^{c} \frac{(-1)^{b}}{2^{2(a+b+j)}} C(b+1, a+j-1) \text {. }
\end{aligned}
$$

We used $p_{+}(\underbrace{1, \ldots, 1}_{2 a+1}, \underbrace{2, \ldots, 2}_{b-1}, 1)=b$ and $p_{+}(\underbrace{1, \ldots, 1}_{2 a+1}, \underbrace{2, \ldots, 2}_{b}, \underbrace{1, \ldots, 1}_{i})=b+1$ for $i \geq 1$.

On the other hand, $p_{-}(\alpha)=b$, so by (63),

$$
\left(\zeta^{-1}\right)_{-}\left(F_{\alpha}\right)=\frac{(-1)^{2(a+b+c)+1+b}}{2^{2(a+b+c)}} C(b, a+c) .
$$

Equating $\left(\zeta^{-1}\right)_{-}\left(F_{\alpha}\right)$ to $\left(\zeta_{+} \zeta^{-1}\right)\left(F_{\alpha}\right)$ gives (65).

A few special cases of Corollary 8.8 are worth stating. We obtain formulas expressing a central binomial coefficient or a Catalan number in terms of bivariate Catalan numbers.

Corollary 8.9. For any non-negative integers $b$ and $c$,

$$
\begin{aligned}
& \left(\begin{array}{c}
2 b \\
b
\end{array}\right)=\frac{1}{4^{c}} C(b, c)+\sum_{j=1}^{c} \frac{1}{4^{j}} C(b+1, j-1), \\
& 2 C(b)=\frac{1}{4^{c}} C(b, c+1)+\sum_{j=1}^{c} \frac{1}{4^{j}} C(b+1, j) .
\end{aligned}
$$

Proof. These follow by choosing $a=0$ and $a=1$ in (65).

Corollary 8.10. Let $H(a, b, c):=C(a, b+c)-C(b, a+c)$. Then

$$
\frac{1}{4^{c}} H(a, b, c)=\sum_{j=1}^{c} \frac{1}{4^{j}} H(b+1, a+1, j-2) .
$$

Proof. This follows from since $C(a, b)=C(b, a)$. 


\section{Appendix. Bivariate Catalan numbers as binomial co- efficients}

Using the easily verified formula

$$
C(m, n)=(-1)^{n} 4^{m+n}\left(\begin{array}{c}
m-1 / 2 \\
m+n
\end{array}\right)
$$

one may restate all results in this paper in terms of binomial coefficients. This allows for simplifications in some of the formulas. We list here our main results in this notation. Let $\alpha=\left(a_{1}, \ldots, a_{k}\right)$ be a composition of $n$.

$$
\begin{aligned}
& \zeta_{-}\left(M_{\alpha}\right)= \begin{cases}(-1)^{k_{e}(\alpha)+\left\lfloor\frac{k_{o}(\alpha)}{2}\right\rfloor}\left(\begin{array}{c}
-1 / 2 \\
\left\lfloor k_{o}(\alpha) / 2\right\rfloor
\end{array}\right) & \text { if } a_{k} \text { is odd } \\
0 & \text { if } a_{k} \text { is even; }\end{cases}
\end{aligned}
$$

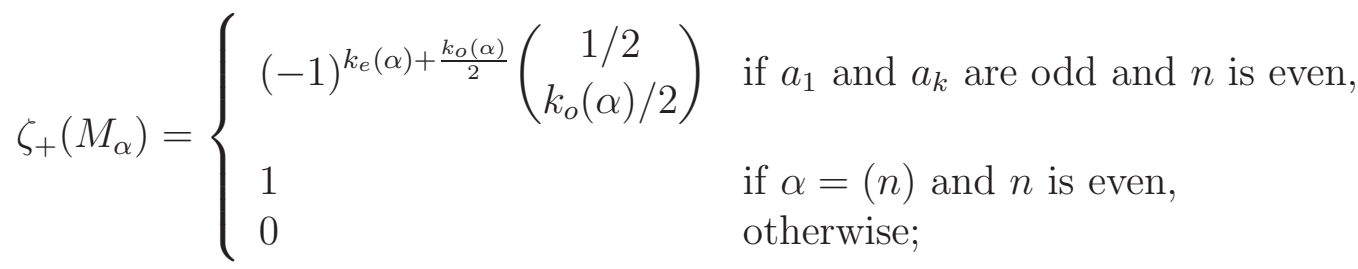

$$
\begin{aligned}
& \zeta_{-}\left(F_{\alpha}\right)=(-1)^{\left\lfloor\frac{n}{2}\right\rfloor}\left(\begin{array}{c}
p_{-}(\alpha)-1 / 2 \\
\lfloor n / 2\rfloor
\end{array}\right) \\
& \zeta_{+}\left(F_{\alpha}\right)= \begin{cases}(-1)^{\frac{n}{2}}\left(\begin{array}{c}
p_{+}(\alpha)-1 / 2 \\
n / 2
\end{array}\right) & \text { if } n \text { is even, } \\
0 & \text { if } n \text { is odd; }\end{cases} \\
& \left(\zeta^{-1}\right)_{-}\left(M_{\alpha}\right)= \begin{cases}(-1)^{k(\alpha)+\left\lfloor\frac{k_{o}(\alpha)}{2}\right\rfloor}\left(\begin{array}{c}
-1 / 2 \\
\left\lfloor k_{o}(\alpha) / 2\right\rfloor
\end{array}\right) & \text { if } a_{1} \text { is odd, } \\
0 & \text { if } a_{1} \text { is even; }\end{cases}
\end{aligned}
$$

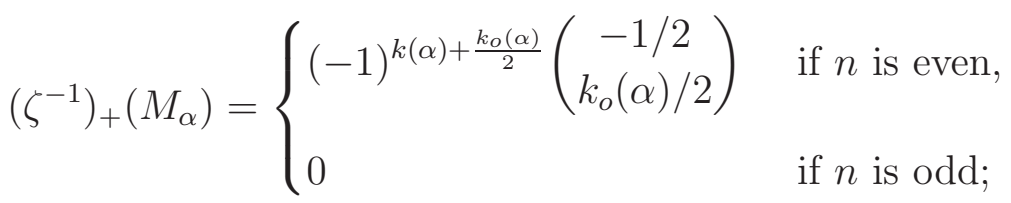

$$
\begin{aligned}
& \left(\zeta^{-1}\right)_{-}\left(F_{\alpha}\right)=(-1)^{\left\lfloor\frac{n+1}{2}\right\rfloor}\left(\begin{array}{c}
p_{-}(\tilde{\alpha})-1 / 2 \\
\lfloor n / 2\rfloor
\end{array}\right) ; \\
& \left(\zeta^{-1}\right)_{+}\left(F_{\alpha}\right)= \begin{cases}(-1)^{\frac{n}{2}}\left(\begin{array}{c}
p_{+}\left(\alpha^{\prime}\right)-1 / 2 \\
n / 2
\end{array}\right) & \text { if } n \text { is even, } \\
0 & \text { if } n \text { is odd } .\end{cases}
\end{aligned}
$$


We mention that the convolution powers of the universal character are

$$
\begin{gathered}
\zeta^{m}\left(M_{\alpha}\right)=\left(\begin{array}{c}
m \\
k(\alpha)
\end{array}\right), \\
\zeta^{m}\left(F_{\alpha}\right)=\left(\begin{array}{c}
m+n-k(\alpha) \\
n
\end{array}\right),
\end{gathered}
$$

for any integer $m[2$, Formula (4.4)].

\section{References}

[1] Marcelo Aguiar, Nantel Bergeron, and Kathryn Nyman, The peak algebra and the descent algebras of types B and D, Trans. Amer. Math. Soc. 356 (2004), 2781-2824.

[2] Marcelo Aguiar, Nantel Bergeron, and Frank Sottile, Combinatorial Hopf algebras and generalized Dehn-Sommerville relations, to appear in Compos. Math., math.CO/0310016.

[3] Marcelo Aguiar and Frank Sottile, Structure of the Malvenuto-Reutenauer Hopf algebra of permutations, Adv. Math. 191 no. 2 (2005), 225-275.

[4] Eugène Catalan, Sur quelques questions relatives aux fonctions elliptiques, Seconde Note. Présentée à l'Académie pontificale des Nuovi Lincei dans la séance du 19 Janvier 1873. Published separately from the main series in size $8^{\circ}, 15 \mathrm{pp}$.

[5] Eugène Catalan, Question 1135, Nouvelles annales de mathématiques: Journal des candidats aux écoles polytechniques et normales, Series 2, 13 (1874), 207.

[6] Eugène Catalan, Mélanges Mathématiques, Tome II, Bruxelles, F. Hayez, 1887. Published also in Extrait des Mémoires de la société royale des sciences de Liège, 2e sér. , XIII, Paris, Gauthier-Villars, 1885.

[7] Eugène Catalan, Mémoire sur quelques décompositions en carrés, Atti dell'Accademia Pontificia Romana de Nuovi Lincei, v. XXXVII, sessione I (1883), 49-114.

[8] Richard Ehrenborg, On posets and Hopf algebras, Adv. Math. 119 (1996), no. 1, 1-25.

[9] Ömer Ĕ̆ecioğlu and Alastair King, Random walks and Catalan factorization, Proceedings of the Thirtieth Southeastern International Conference on Combinatorics, Graph Theory, and Computing (Boca Raton, FL, 1999). Congr. Numer. 138 (1999), 129-140.

[10] F. Fares, Quelques constructions d'algèbres et de coalgèbres, Université du Québec à Montréal (1999). 
[11] Ira Gessel, Multipartite P-partitions and products of skew Schur functions, in Combinatorics and Algebra (Boulder, Colo., 1983), C. Greene, ed., vol. 34 of Contemp. Math. AMS (1984) pp. 289-317.

[12] Ira Gessel, Super ballot numbers, J. Symbolic Comput. 14 (1992), no. 2-3, 179-194.

[13] Ira Gessel, private communication (2004).

[14] Henry W. Gould, Combinatorial identities. A standardized set of tables listing 500 binomial coefficient summations. Henry W. Gould, Morgantown, W. Va., 1972.

[15] Michiel Hazewinkel, Quasi-symmetric functions, Formal power series and algebraic combinatorics (Moscow, 2000), 30-44, Springer, Berlin, 2000.

[16] Michael E. Hoffman, Quasi-shuffle products, J. Algebraic Combin. 11 (2000), no. 1, 49-68.

[17] Claudia Malvenuto, Produits et coproduits des fonctions quasi-symétriques et de l'algèbre des descents, no. 16, Laboratoire de combinatoire et d'informatique mathématique (LACIM), Univ. du Québec à Montréal, Montréal, 1994.

[18] Karol A. Penson and J.-M. Sixdeniers, Integral representations of Catalan and related numbers, Journal of Integer Sequences, Vol. 4 (2001), Article 01.2.5 (6 pages).

[19] John Riordan, Combinatorial identities. John Wiley \& Sons, New York, 1968.

[20] Richard Stanley, Ordered structures and partitions, Mem. Amer. Math. Soc. 119 (1972).

[21] Richard Stanley, Enumerative combinatorics. Vol. 2, Cambridge University Press, Cambridge, 1999, With a foreword by Gian-Carlo Rota and appendix 1 by Sergey Fomin.

[22] Richard Stanley, Catalan addendum, version of 22 December 2004, 46 pages. http://www-math.mit.edu/ rstan/ec/catadd.pdf

[23] John Stembridge, Enriched P-partitions, Trans. AMS 349 (1997) 763-788.

[24] Jean-Yves Thibon and B.-C.-V. Ung, Quantum quasi-symmetric functions and Hecke algebras, J. Phys. A 29 (1996), no. 22, 7337-7348.

[25] Koloman von Szily, Über die quadratsummen der binomialcoefficienten, Mathematische und naturwissenschaftliche Berichte aus Ungarn (Ungar. Ber.) 12 (1893), 84-91. 